\title{
Averaging over Narain moduli space
}

\author{
Alexander Maloney ${ }^{a}$ and Edward Witten ${ }^{b}$ \\ ${ }^{a}$ Department of Physics, McGill University, \\ Montreal, QC H3A 2T8, Canada \\ ${ }^{b}$ Institute for Advanced Study, \\ Einstein Drive, Princeton, NJ 08540, U.S.A. \\ E-mail: alex.maloney@mcgill.ca, witten@ias.edu
}

ABSTRACT: Recent developments involving JT gravity in two dimensions indicate that under some conditions, a gravitational path integral is dual to an average over an ensemble of boundary theories, rather than to a specific boundary theory. For an example in one dimension more, one would like to compare a random ensemble of two-dimensional CFT's to Einstein gravity in three dimensions. But this is difficult. For a simpler problem, here we average over Narain's family of two-dimensional CFT's obtained by toroidal compactification. These theories are believed to be the most general ones with their central charges and abelian current algebra symmetries, so averaging over them means picking a random CFT with those properties. The average can be computed using the Siegel-Weil formula of number theory and has some properties suggestive of a bulk dual theory that would be an exotic theory of gravity in three dimensions. The bulk dual theory would be more like $\mathrm{U}(1)^{2 D}$ Chern-Simons theory than like Einstein gravity.

Keywords: AdS-CFT Correspondence, Conformal Field Theory, Gauge-gravity correspondence

ArXiv ePrint: 2006.04855 


\section{Contents}

1 Introduction $\quad 1$

2 The Siegel-Weil formula in genus one $\quad 7$

2.1 A practice case: $D=1 \quad 7$

2.2 The Siegel-Weil formula for higher $D \quad 9$

2.3 Gravitational interpretation of the formula 11

2.4 Adding more information about the CFT 13

3 Higher genus and disconnected boundaries 18

$\begin{array}{lll}3.1 & \text { Higher genus } & 18\end{array}$

3.2 Disconnected boundaries 21

3.3 Genus zero 26

4 Path integrals in gauge theory $\quad 27$

$\begin{array}{lll}4.1 & \text { Preliminaries } & 27\end{array}$

$\begin{array}{lll}4.2 & \text { Path integral on a handlebody } & 29\end{array}$

4.3 Disconnected boundaries 33

$4.4 \mathrm{U}(1)^{2 D}$ and $\mathbb{R}^{2 D}$ Chern-Simons theories 35

A Derivation of the Siegel-Weil formula at $D>1$ and $g>1 \quad 37$

A.1 Narain moduli space $\quad 37$

$\begin{array}{lll}\text { A.2 Geometry of Siegel upper half space } & 38\end{array}$

A.3 The average CFT partition function at genus $g \quad 40$

\section{Introduction}

A simple model of gravity in two dimensions - JT gravity — is dual to a random ensemble of quantum mechanical systems, rather than a specific quantum mechanical system [1]. It is natural to wonder if something similar happens in higher dimensions. For example, gravity is still relatively simple in three spacetime dimensions, at least from some points of view. Are there simple theories of gravity in three dimensions - maybe even pure Einstein gravity - that are dual in some sense to a random two-dimensional conformal field theory (CFT)?

The difficulty here is that while a quantum mechanical system can be defined by specifying a Hamiltonian, the data required to specify a 2d CFT are far more complicated. Accordingly, it is far from clear what should be meant by a random $2 \mathrm{~d}$ CFT, though one can possibly get some insight from results about asymptotic behavior of dimensions and 
couplings of CFT primaries [2-5]. It is also not clear what should be the partition function of pure Einstein gravity, though there have been a number of attempts [6-8].

Here we will consider a simpler problem. We consider 2d CFT's with left and right central charges $\left(c_{\ell}, c_{r}\right)=(D, D)$ (for some positive integer $D$ ) that also have left- and right-moving $\mathrm{U}(1)^{D}$ current algebras. It is expected that any such theory is in the family originally constructed by Narain $[9,10]$, the parameter space being the locally symmetric space

$$
\mathcal{M}_{D}=\mathrm{SO}(D, D ; \mathbb{Z}) \backslash \mathrm{SO}(D, D ; \mathbb{R}) / \mathrm{SO}(D) \times \mathrm{SO}(D) .
$$

(Here $\mathrm{SO}(D, D ; \mathbb{Z})$ must be understood as the automorphism group of an even integer unimodular lattice $\Lambda$ of signature $(D, D)$.) As a CFT moduli space, $\mathcal{M}_{D}$ carries a natural Zamolodchikov metric, which determines a natural measure. This is actually the same metric and measure that $\mathcal{M}_{D}$ gets because it is locally homogeneous, that is, it is the quotient of the homogeneous space $\mathrm{SO}(D, D ; \mathbb{R}) / \mathrm{SO}(D) \times \mathrm{SO}(D)$ by the discrete group $\mathrm{SO}(D, D ; \mathbb{Z}) . \mathcal{M}_{D}$ has finite measure for any $D>1$, and when this is the case, it makes sense to average over $\mathcal{M}_{D}$ in its natural measure. This is what we will mean by "averaging over Narain moduli space."

For a point $m \in \mathcal{M}_{D}$, let $Z_{\Sigma}(m, \tau)$ be the partition function of the corresponding CFT on a Riemann surface $\Sigma$ with modular parameters $\tau$. The lattice sum that controls the $m$ dependence of $Z_{\Sigma}(m, \tau)$ is a nonholomorphic theta function that was originally introduced by C. L. Siegel and rediscovered by Narain; we will call it the Siegel-Narain theta function, and denote it as ${ }^{1} \Theta(m, \tau)$. It turns out that the average of $\Theta(m, \tau)$ over $m \in \mathcal{M}_{D}$ can be computed in a simple way, using what is known in number theory as the Siegel-Weil formula, developed by Siegel, Maass, and Weil [11-15]. The Siegel-Weil formula expresses the average over $m$ of $\Theta(m, \tau)$ in terms of a non-holomorphic Eisenstein series $E_{D / 2}(\tau)$ with modular weights $(D / 2, D / 2) .^{2}$

If the ensemble of Narain theories is dual to a theory of gravity, that theory is not going to be a conventional one. First of all, since the CFT's considered have U $(1)^{2 D}$ current algebra and in particular $\mathrm{U}(1)^{2 D}$ global symmetry, the bulk theory will have $\mathrm{U}(1)^{2 D}$ gauge symmetry. The perturbative anomalies of the boundary current algebra become ChernSimons couplings in the bulk theory. Those anomalies are controlled by the even integer unimodular lattice $\Lambda_{I J}, I, J=1, \cdots, 2 D$ that is used in constructing the CFT. Thus at a minimum we expect the bulk theory to have gauge fields $A^{I}, I=1, \cdots, 2 D$ of the group $\mathrm{U}(1)^{2 D}$ with Chern-Simons couplings. ${ }^{3}$ On a three-manifold $Y$, the Chern-Simons action is

$$
I_{\mathrm{CS}}=\sum_{I, J} \frac{\Lambda_{I J}}{2 \pi} \int_{Y} A^{I} \wedge \mathrm{d} A^{J} .
$$

\footnotetext{
${ }^{1} \Theta(m, \tau)$ is not holomorphic or anti-holomorphic in $\tau$. To emphasize this, one could denote it as $\Theta(m, \tau, \bar{\tau})$. To lighten notation, we will not do that.

${ }^{2}$ Non-holomorphic or real analytic Eisenstein series may be less familiar than holomorphic ones; however, they appear in the effective action of string theory [16, 17]. The constant term in the Siegel-Weil formula, which is known as the Smith-Minkowski-Siegel mass formula, computes volumes of moduli spaces and has appeared studies of the moduli space of conformal field theories [18, 19].

${ }^{3} \mathrm{~A}$ variant that we consider in section 4.4 is that the gauge group is really $\mathbb{R}^{2 D}$.
} 


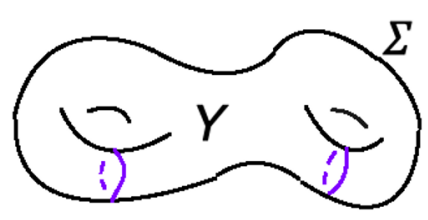

Figure 1. If a closed Riemann surface $\Sigma$ is embedded in $\mathbb{R}^{3}$ in an arbitrary fashion, then its "interior" is, topologically, a handlebody $Y$. Such an embedding of $\Sigma$ determines a distinguished sublattice $\Gamma_{0}$ of the first homology $\Gamma=H_{1}(\Sigma, \mathbb{Z})$, spanned by one-cycles that are contractible in $Y$. In the present example, $\Sigma$ has genus 2 , and $\Gamma_{0}$ is spanned by the two one-cycles drawn.

The Narain CFT's require that $\Sigma$ should be oriented (because the target space $B$-field plays an important role). To define the Chern-Simons action, $Y$ should be oriented, in such a way that along the boundary its orientation induces the orientation of $\Sigma=\partial Y$.

The action (1.2) is diffeomorphism-invariant without any need for a metric tensor. One may think that to get the dual theory we want, we must add a metric tensor and a gravitational action. But there are reasons to believe that this is not the case. In the boundary theory, the stress tensor can be expressed in terms of the currents via the Sugawara construction: if $T$ is the holomorphic stress tensor, and $J^{a}, a=1, \cdots, D$ are the holomorphic currents, the formula is $T(z)=\sum_{a}: J^{a} J^{a}(z):$. What is the bulk dual of the Sugawara formula? It is plausible that the bulk dual of the fact that the stress tensor is a function of the currents rather than being "new" is that we should not introduce in bulk a metric tensor that is independent of the gauge fields. Thus we might hope that the bulk dual of the average over Narain moduli space is simply the gauge theory with action $I_{\mathrm{CS}}$, or at least, something more like this than a theory with a dynamical metric tensor. This has to be supplemented with a recipe for what $Y$ should be. The Chern-Simons gauge theory per se does not suggest any specific rule to sum over $Y$ 's with fixed conformal boundary $\Sigma$; in fact, the Chern-Simons path integral on a three-manifold $Y$ makes sense for any particular $Y$, and the Chern-Simons gauge theory does not come with any rationale for summing over choices of $Y$.

However, the Siegel-Weil formula suggests how to sum over $Y$ as long as $\Sigma$ is connected. Topologically, the simplest class of three-manifolds with boundary $\Sigma$ are "handlebodies." An orientable two-manifold $\Sigma$ can be embedded in $\mathbb{R}^{3}$ (in many topologically distinct ways). Any such embedding divides the complement of $\Sigma$ in $\mathbb{R}^{3}$ into two components; the "interior" component is called a handlebody (figure 1). With a plausible recipe for how to compute $Z_{Y}(\tau)$ for $Y$ a handlebody, the Siegel-Weil formula gives

$$
\left\langle Z_{\Sigma}(m, \tau)\right\rangle=\sum_{Y \in \mathcal{J}} Z_{Y}(\tau)
$$

where \langle\rangle represents an average over $\mathcal{M}_{D}$, and $\mathcal{J}$ is the set of all handlebodies with boundary $\Sigma$.

In pure Einstein gravity with negative cosmological constant, if $\Sigma$ has genus 1 , there is a semi-plausible justification for summing only over handlebodies [6]. Any three-manifold 
that is a classical solution of Einstein gravity with negative cosmological constant and that has a conformal boundary consisting of a single component of genus 1 is a handlebody. Therefore, if the path integral of Euclidean quantum gravity should be constructed as an expansion around critical points (or as an integral over Lefschetz thimbles associated to critical points), then only handlebodies can contribute if $\Sigma$ has genus 1. (In a supersymmetric extension of three-dimensional gravity, there can be a more clear-cut justification to sum only over handlebodies in evaluating an appropriate index [20].)

This justification to include only handlebodies is not entirely convincing for pure Einstein gravity, but in any case, we are here not considering pure Einstein gravity, but a more exotic theory that can be approximated by the Chern-Simons theory (1.2). Moreover, even in pure Einstein gravity, for $\Sigma$ of genus $g>1$, there are classical solutions with conformal boundary $Y$ that are not handlebodies; see [21].

We can get, in a sense, a clearer picture of what the sum in eq. (1.3) would have to mean by considering the case that $\Sigma$ is not connected. If $\Sigma$ is the disjoint union of components $\Sigma_{\alpha}, \alpha=1, \cdots, s$, with modular parameters $\tau_{\alpha}$, then for fixed $m \in \mathcal{M}_{D}$, the partition function is a simple product: $Z_{\Sigma}(m, \tau)=\prod_{\alpha} Z_{\Sigma_{\alpha}}\left(m, \tau_{\alpha}\right)$ (here we write $\tau$ for the whole collection of all the $\tau_{\alpha}$ ). After averaging over $m$, this is of course no longer true:

$$
\left\langle Z_{\Sigma}(m, \tau)\right\rangle \neq \prod_{\alpha}\left\langle Z_{\Sigma_{\alpha}}\left(m, \tau_{\alpha}\right)\right\rangle .
$$

The Siegel-Weil formula gives an answer for $\left\langle Z_{\Sigma}(m, \tau)\right\rangle$ also when $\Sigma$ is not connected, but to describe it, we have to first restate the formula in the connected case. Let $\Sigma$ be a connected Riemann surface of genus $g$. The first homology group $H_{1}(\Sigma, \mathbb{Z})$ is a lattice $\Gamma \cong \mathbb{Z}^{2 g}$. For $\gamma, \gamma^{\prime} \in H_{1}(\Sigma, \mathbb{Z})$, we denote their oriented intersection number as $\left\langle\gamma, \gamma^{\prime}\right\rangle$. The pairing $\langle$,$\rangle is antisymmetric and nondegenerate. A "Lagrangian sublattice" \Gamma_{0} \subset H_{1}(\Sigma, \mathbb{Z})$ is a primitive ${ }^{4}$ sublattice of rank $g$ on which the intersection pairing vanishes; in other words, $\left\langle\gamma, \gamma^{\prime}\right\rangle=0$ for $\gamma, \gamma^{\prime} \subset \Gamma_{0}$. For example, any set of $A$-cycles on $\Sigma$ determines a Lagrangian sublattice, namely the sublattice of $H_{1}(\Sigma, \mathbb{Z})$ spanned by the homology classes of those $A$-cycles. Alternatively, any oriented manifold $Y$ with boundary $\Sigma$ determines a Lagrangian sublattice, namely the sublattice $\Gamma_{0} \subset H_{1}(\Sigma, \mathbb{Z})$ that is spanned by all onecycles $\gamma \subset \Sigma$ that are boundaries of two-manifolds in $Y$.

If $\Sigma$ is connected, then every Lagrangian sublattice $\Gamma_{0}$ is associated in this way to a distinguished three-manifold $Y$, namely a handlebody. (To construct $Y$, pick a set of $A$ cycles or disjoint simple closed curves in $\Sigma$ that provides a basis of $\Gamma_{0}$, and embed $\Sigma$ in $\mathbb{R}^{3}$ so that these $A$-cycles are contractible in the interior.) This gives a one-to-one correspondence between handlebodies and associated Lagrangian sublattices. However, associated to the same sublattice there are infinitely many other three-manifolds that are not handlebodies.

For connected $\Sigma$, using the correspondence between handlebodies and Lagrangian sublattices of $H_{1}(\Sigma, \mathbb{Z})$, we can reinterpret $\mathcal{J}$ as the set of Lagrangian sublattices. Once this

\footnotetext{
${ }^{4}$ Here "primitive" means that if $\Gamma_{0}$ contains a nonzero multiple of some $x \in H_{1}(\Sigma, \mathbb{Z})$, then it actually contains $x$. In other words, we are not allowed to replace $\Gamma_{0}$ by a proper sublattice of the same rank. An equivalent definition is that a sublattice $\Gamma_{0} \subset H_{1}(\Sigma, \mathbb{Z})$ is primitive if the quotient $H_{1}(\Sigma, \mathbb{Z}) / \Gamma_{0}$ is torsionfree. All sublattices considered in this paper are assumed to be primitive; this condition is not always stated.
} 
is done, the Siegel-Weil formula says that (with a plausible interpretation of $Z_{Y}(\tau)$ ) the formula (1.3) holds for all $\Sigma$, connected or not. The difference is that if $\Sigma$ is not connected, there is no distinguished choice of a manifold $Y$ associated with a given Lagrangian sublattice. So to state the formula in a way that is valid whether $\Sigma$ is connected or not, we have to interpret the sum on the right hand side of eq. (1.3) as a sum over Lagrangian sublattices. We write it as such:

$$
\left\langle Z_{\Sigma}(m, \tau)\right\rangle=\sum_{\Gamma_{0}} Z_{\Gamma_{0}}(\tau),
$$

where the sum now runs over the set of Lagrangian sublattices and $Z_{\Gamma_{0}}(\tau)$ is the contribution to the partition function associated to the Lagrangian sublattice $\Gamma_{0}$.

This tells us something about the exotic gravitational theory that is dual to an average over Narain moduli space - if such a theory exists. In this theory, the objects that are analogous to "manifolds with boundary $\Sigma$ " in ordinary geometry are classified by Lagrangian sublattices of $H_{1}(\Sigma, \mathbb{Z})$. Apparently, the additional topological invariants that would be present in ordinary geometry are lacking in this more exotic theory.

A conceivable alternative interpretation might be that in the exotic theory, there are many "manifolds" associated to a given Lagrangian sublattice $\Gamma_{0}$, and the sum over all of these is giving what we call $Z_{\Gamma_{0}}(\tau)$. This seems less plausible, in part for the following reason. We will see that to reproduce the effect of averaging over $\mathcal{M}_{D}$, we have to use $Z_{\Gamma_{0}}(\tau)=c_{\Gamma_{0}}^{D}$, where $c_{\Gamma_{0}}$ depends on $\Gamma_{0}$ and $\tau$ but not on $D$. If $Z_{\Gamma_{0}}(\tau)$ is built in a nontrivial way as a sum of contributions of different "manifolds" $\Gamma_{0, i}$, then, since a nontrivial formula $c_{\Gamma_{0}}^{D}=\sum_{i} c_{\Gamma_{0, i}}^{D}$ is not going to hold for all $D$ no matter what we assume for the $c_{\Gamma_{0, i}}$, individual contributions must have a more complicated dependence on $D$, which then cancels out in the sum $\sum_{i} c_{\Gamma_{0, i}}^{D}$. It seems more economical to assume that in the exotic theory under discussion, the analog of a "manifold with boundary $\Sigma$ " is just classified by the choice of $\Gamma_{0}$. This possibility does not sound completely far-fetched; it would be in keeping with the idea that among all the topological invariants of classical geometry, only some that are particularly robust are well-defined in quantum gravity.

A close cousin of the $\mathrm{U}(1)^{2 D}$ current algebra considered in the present paper is a rational conformal field theory (RCFT), with an extended chiral algebra that has only finitely many modules. Attempts have been made to express RCFT partition functions in terms of sums over modular images of a function which would be hypothetically the partition function of an exotic theory of gravity on a handlebody [22, 23]. This program is successful at $c=1 / 2$ at least in the sense that a suitable function exists, except that one has to assume slightly puzzling equivalences between different handlebodies. The program does not seem to work in the same way for theories with $1 / 2<c<1$. Those theories have a chiral algebra that admits more than one modular-invariant partition function. It may be that the bulk path integral in these cases represents a sort of average over the possible boundary theories, given a knowledge of the chiral algebra. That is in the spirit of what we find here for a boundary theory with $\mathrm{U}(1)^{D}$ left-moving and right-moving current algebras. The difference is that instead of finitely many possible boundary theories with a given chiral algebra, we will have a continuous family of possible boundary theories, parametrized by $\mathcal{M}_{D}$. 
The organization of this article is as follows. Section 2 is devoted to averaging over Narain moduli space in the case of a surface $\Sigma$ of genus 1. The Siegel-Weil formula that carries out this averaging is explained in sections 2.1 and 2.2. In section 2.3 , we attempt to interpret the output of the Siegel-Weil formula in terms of a bulk dual theory. In section 2.4, we consider what happens if we supply more information about the CFT by for example specifying a particular extension of the boundary current algebra. This leads to a restricted averaging problem that is governed by a more general version of the Siegel-Weil formula. In the most extreme case the average is a sum over a finite set of CFTs, rather than an integral over a moduli space; nevertheless, the result has a plausible gravity interpretation.

In section 3 , we study the generalization to surfaces of higher genus and to the case of a surface with multiple connected components. The Siegel-Weil formula is applicable in any genus. As we have already explained, to interpret the result in terms of a bulk dual theory, we seemingly have to assume that the dual theory has a nonclassical notion of "manifold with boundary $\Sigma$." The Siegel-Weil formula also applies to the case of a surface $\Sigma$ with multiple connected components. Connected correlators between different components would come, in ordinary gravity, from connected manifolds with disconnected boundary, as in the case of JT gravity [1]. We study explicitly one example of a contribution to a connected correlator, which is related to the spectral form factor $\left\langle Z_{T^{2}}\left(m, \tau^{\prime}\right) Z_{T^{2}}\left(m, \tau^{\prime \prime}\right)\right\rangle$. The contribution that we analyze vanishes exponentially for large $D$, compared to the corresponding disconnected correlator. This is as if adding a wormhole that connects two components increases the classical action, which is probably the general state of affairs of a hypothetical theory of gravity related to the Siegel-Weil formula. The contribution to the spectral form factor we analyze is non-zero at large Lorentzian time, a feature which is indicative of theories with a discrete spectrum. We discuss in section 3.3 the special case that $\Sigma$ is of genus 0 or has a component of genus 0 . In averaging over Narain moduli space, a surface of genus 0 has no connected correlator with anything else. In the dual theory, this might mean that there is no way to make a wormhole connecting a surface of genus zero to another surface. An alternative interpretation might be that in the dual theory, there is no notion of whether spacetime is connected.

Finally, in section 4, we describe in more detail the attempt to interpret the dual of the average over Narain moduli space in terms of a gauge theory with gauge group $\mathrm{U}(1)^{2 D}$ or possibly $\mathbb{R}^{2 D}$. We find that when the boundary has a single connected component, bulk Chern-Simons theory on a handlebody, under some assumptions, exactly reproduces the corresponding term in the Siegel-Weil formula. Indeed, there is a sense in which the bulk partition function is one-loop exact in a perturbative expansion in $1 / D .{ }^{5}$ However, we will discover that the situation is considerably more subtle when the boundary is disconnected.

Appendix A contains further details about Narain moduli space, as well as a sketch of the derivation of the Siegel-Weil formula at genus $g>1$ and $D>1$.

When this draft was largely complete, we learned of parallel work by Afkhami-Jeddi, Cohn, Hartman, and Tajdini [25].

\footnotetext{
${ }^{5}$ This is much simpler than the case of pure gravity, where the perturbative expansion is one-loop exact when the boundary is a torus [6] but is not expected to terminate when the boundary has higher genus [24].
} 


\section{The Siegel-Weil formula in genus one}

\subsection{A practice case: $D=1$}

We begin by discussing the Siegel-Weil formula in genus 1, where we can write somewhat more explicit formulas and the hypothetical gravitational dual can be analyzed in a more direct way.

In general, the Narain models are sigma-models with target a $D$-torus $T^{D}$, leading to a CFT with $\left(c_{\ell}, c_{r}\right)=(D, D)$. We will begin with the case $D=1$, to illustrate the main idea. We take the target space to be a circle of circumference $2 \pi R$. The moduli space $\mathcal{M}_{1}$ in this example is parametrized by $R$, which can be restricted to the range $1 \leq R<\infty$ because of the $R \rightarrow 1 / R$ duality symmetry. Parametrizing the circle by an angle-valued field $X$, the action (with conventions as in [26], eq. (2.1.1)) is

$$
I=\frac{R^{2}}{4 \pi \alpha^{\prime}} \int \mathrm{d}^{2} \sigma \partial_{\alpha} X \partial^{\alpha} X
$$

The marginal operator associated with a deformation $R \rightarrow R+\delta R$ is $\frac{R \delta R}{2 \pi \alpha^{\prime}} \partial_{\alpha} X \partial^{\alpha} X$, and its two-point function is proportional to $(\delta R)^{2} / R^{2}$. This gives the Zamolodchikov metric of $\mathcal{M}_{1}$

$$
\mathrm{d} s^{2}=4 \frac{\mathrm{d} R^{2}}{R^{2}}
$$

which is also the natural metric on $\mathcal{M}_{1}$ as a locally homogeneous space. (The factor of 4 is chosen for later convenience and to agree with the more general formula (A.3) of appendix A.)

The partition function of the model on a genus 1 surface $\Sigma$ with modular parameter $\tau=\tau_{1}+\mathrm{i} \tau_{2}$ is (see [26], eq. (8.2.9))

$$
Z_{\Sigma}(R, \tau)=\frac{\Theta(R, \tau)}{|\eta(\tau)|^{2}}
$$

where $\eta(\tau)$ is the Dedekind eta function

$$
\eta(\tau)=q^{1 / 24} \prod_{n=1}^{\infty}\left(1-q^{n}\right), \quad q=\exp (2 \pi \mathrm{i} \tau),
$$

and $\Theta(R, \tau)$ is the $D=1$ case of the Siegel-Narain theta function,

$$
\Theta(R, \tau)=\sum_{n, w \in \mathbb{Z}} Q(n, w ; R, \tau)
$$

with

$$
Q(n, w ; R, \tau)=\exp \left(-\pi \tau_{2}\left(\frac{\alpha^{\prime} n^{2}}{R^{2}}+\frac{w^{2} R^{2}}{\alpha^{\prime}}\right)+2 \pi \mathrm{i} \tau_{1} n w\right) .
$$

The integers $n$ and $w$ are the momentum and winding quantum numbers of a string. 
A small calculation gives

$$
\begin{aligned}
\tau_{2} \frac{\partial}{\partial \tau_{2}} Q & =-\pi \tau_{2}\left(\frac{\alpha^{\prime} n^{2}}{R^{2}}+\frac{w^{2} R^{2}}{\alpha^{\prime}}\right) Q \\
\tau_{2}^{2} \frac{\partial^{2}}{\partial \tau_{2}^{2}} Q & =\pi^{2} \tau_{2}^{2}\left(\frac{\alpha^{\prime} n^{2}}{R^{2}}+\frac{w^{2} R^{2}}{\alpha^{\prime}}\right)^{2} Q \\
\tau_{2}^{2} \frac{\partial^{2}}{\partial \tau_{1}^{2}} Q & =-4 \pi^{2} \tau_{2}^{2} n^{2} w^{2} \\
\left(R \frac{\partial}{\partial R}\right)^{2} Q & =\left(4 \pi^{2} \tau_{2}^{2}\left(\frac{\alpha^{\prime} n^{2}}{R^{2}}-\frac{w^{2} R^{2}}{\alpha^{\prime}}\right)^{2}-4 \pi \tau_{2}\left(\frac{\alpha^{\prime} n^{2}}{R^{2}}+\frac{w^{2} R^{2}}{\alpha^{\prime}}\right)\right) Q .
\end{aligned}
$$

Therefore

$$
\left(\tau_{2}^{2}\left(\frac{\partial^{2}}{\partial \tau_{1}^{2}}+\frac{\partial^{2}}{\partial \tau_{2}^{2}}\right)+\tau_{2} \frac{\partial}{\partial \tau_{2}}-\frac{1}{4}\left(R \frac{\partial}{\partial R}\right)^{2}\right) Q=0,
$$

and it follows immediately that $\Theta$ obeys the same equation:

$$
\left(\tau_{2}^{2}\left(\frac{\partial^{2}}{\partial \tau_{1}^{2}}+\frac{\partial^{2}}{\partial \tau_{2}^{2}}\right)+\tau_{2} \frac{\partial}{\partial \tau_{2}}-\frac{1}{4}\left(R \frac{\partial}{\partial R}\right)^{2}\right) \Theta(R, \tau)=0 .
$$

The measure on $\mathcal{M}_{1}$ can be deduced from the metric (2.2) and is

$$
\mu(R)=\frac{\mathrm{d} R}{2 R}
$$

The volume of $\mathcal{M}_{1}$ is infinite, so averaging over $\mathcal{M}_{1}$ does not make sense, as remarked in the introduction. Let us ignore this for a moment and explain the strategy in the derivation of the Siegel-Weil formula. We would define a function $F_{1}(\tau)$ by integrating $\Theta(R, \tau)$ over $\mathcal{M}_{1}$ :

$$
F_{1}(\tau)=\int_{1}^{\infty} \frac{\mathrm{d} R}{2 R} \Theta(R, \tau) .
$$

In reality, this integral does not converge, since $\Theta \sim R$ for $R \rightarrow \infty$. Then using the differential equation for $\Theta$, we find that

$$
\begin{aligned}
\left(\tau_{2}^{2}\left(\frac{\partial^{2}}{\partial \tau_{1}^{2}}+\frac{\partial^{2}}{\partial \tau_{2}^{2}}\right)+\tau_{2} \frac{\partial}{\partial \tau_{2}}\right) F_{1}(\tau) & =\frac{1}{8} \int_{1}^{\infty} \frac{\mathrm{d} R}{R}\left(R \frac{\partial}{\partial R}\right)^{2} \Theta(R, \tau) \\
& =\frac{1}{8} \int_{1}^{\infty} \mathrm{d} R \frac{\partial}{\partial R}\left(R \frac{\partial \Theta(R, \tau)}{\partial R}\right) .
\end{aligned}
$$

Next one tries to integrate by parts to prove the vanishing of the right hand side of this equation. There is no surface term at $R=1$ because the $R \rightarrow 1 / R$ symmetry ensures that $\left.\partial_{R} \Theta\right|_{R=1}=0$. If there were also no surface term at $R=\infty$, we would deduce a differential equation for $F_{1}(\tau)$ :

$$
\left(\tau_{2}^{2}\left(\frac{\partial^{2}}{\partial \tau_{1}^{2}}+\frac{\partial^{2}}{\partial \tau_{2}^{2}}\right)+\tau_{2} \frac{\partial}{\partial \tau_{2}}\right) F_{1}(\tau)=0
$$

The derivation of the Siegel-Weil formula would be completed by using this differential equation together with a knowledge of the behavior for $\tau_{2} \rightarrow \infty$ to determine $F_{1}(\tau)$. 
The only problem with this derivation is that the behavior for $R \rightarrow \infty$ does not allow either the definition of $F_{1}(\tau)$ in eq. (2.11) or the integration by parts that would show the vanishing of eq. (2.12). Hence we will move on to the case of larger $D$, where such a derivation does work.

Before doing so, we restate the differential equation in a convenient form. The Laplacian of $\mathcal{M}_{1}$ in the metric $(2.2)$ is

$$
\Delta_{\mathcal{M}_{1}}=-\frac{1}{4}\left(R \frac{\mathrm{d}}{\mathrm{d} R}\right)^{2} .
$$

The natural metric of the upper half plane $\mathcal{H}$ is

$$
\mathrm{d} s^{2}=\frac{\mathrm{d} \tau_{1}^{2}+\mathrm{d} \tau_{2}^{2}}{\tau_{2}^{2}}
$$

Acting on a scalar function, the Laplacian of the upper half plane is

$$
\Delta_{\mathcal{H}}=-\tau_{2}^{2}\left(\frac{\partial^{2}}{\partial \tau_{1}^{2}}+\frac{\partial^{2}}{\partial \tau_{2}^{2}}\right)
$$

Therefore the differential equation for the Siegel-Narain theta function for $D=1$ can be written

$$
\left(\Delta_{\mathcal{H}}-\tau_{2} \frac{\partial}{\partial \tau_{2}}-\Delta_{\mathcal{M}_{1}}\right) \Theta(R, \tau)=0
$$

\subsection{The Siegel-Weil formula for higher $D$}

For general $D$, we consider a sigma-model with target $T^{D}$ and general (constant) metric $G$ and two-form field $B$. $G$ and $B$ together are the moduli that parametrize $\mathcal{M}_{D}$; we schematically denote these moduli as $m$. For $D>1, \mathcal{M}_{D}$ has finite volume ${ }^{6}$ and we normalize its measure $\mu(m)$ so that the volume is 1 :

$$
\int_{\mathcal{M}_{D}} \mathrm{~d} \mu(m)=1
$$

The partition function of the sigma-model in genus 1 is

$$
Z_{\Sigma}(m, \tau)=\frac{\Theta(m, \tau)}{|\eta(\tau)|^{2 D}}
$$

where $\Theta(m, \tau)$ is the Siegel-Narain theta function that comes from a sum over momenta and windings. See eq. (A.5) for the explicit formula.

\footnotetext{
${ }^{6}$ For example, for $D=2$, using the relationships $\mathrm{SO}(2,2, \mathbb{R}) \cong(\mathrm{SL}(2, \mathbb{R}) \times \mathrm{SL}(2, \mathbb{R})) / \mathbb{Z}_{2}$ and $\mathrm{SO}(2,2, \mathbb{Z}) \cong$ $(\mathrm{SL}(2, \mathbb{Z}) \times \mathrm{SL}(2, \mathbb{Z})) / \mathbb{Z}_{2}$, one can show that $\mathcal{M}_{2}$ is $($ calF $\times \mathcal{F}) / \mathbb{Z}_{2}$, where $\mathcal{F} \equiv \operatorname{SL}(2, \mathbb{Z}) \backslash \mathcal{H}$, with $\mathcal{H}=$ $\mathrm{SL}(2, \mathbb{R}) / \mathrm{U}(1)$ being the upper half plane. $\mathcal{F}$ is isomorphic to the moduli space of Riemann surfaces of genus 1 and has finite volume. Using the metric (A.3), one can show that as $D$ increases, the volume integral converges more rapidly. The same is true for the integral in (2.21). To verify the last statement, one has to take into account the behavior of the function $\Theta$ near infinity in the space of target space metrics.
} 
$\Theta(m, \tau)$ obeys a differential equation that generalizes eq. (2.17) for $d=1$ :

$$
\left(\Delta_{\mathcal{H}}-D \tau_{2} \frac{\partial}{\partial \tau_{2}}-\Delta_{\mathcal{M}_{D}}\right) \Theta(R, \tau)=0
$$

A derivation of this equation similar to the one in section 2.1 is sketched in appendix A.

The next step is to average $\Theta(m, \tau)$ over the Narain moduli space $\mathcal{M}_{D}$, defining

$$
F_{D}(\tau)=\int_{\mathcal{M}_{D}} \mathrm{~d} \mu(m) \Theta(m, \tau)
$$

Actually, this integral converges only for $D>2$, so in continuing, we make that restriction. $\left(\mathcal{M}_{2}\right.$ has finite volume, but $\Theta(m, \tau)$ grows at infinity in such a way that the integral is divergent for $D=2$. To be precise, $\Theta(m, \tau)$ grows in the limit that the target space volume is large and also in the "large complex structure" limit.) Following the same steps as in section 2.1, we deduce from the last two formulas a differential equation for $F_{D}(\tau)$ :

$$
\left(\Delta_{\mathcal{H}}-D \tau_{2} \frac{\partial}{\partial \tau_{2}}\right) F_{D}(\tau)=0
$$

In addition to satisfying this differential equation, $F_{D}(\tau)$ transforms under modular transformations with weights ${ }^{7}(D / 2, D / 2)$, since $F_{D}(\tau) /|\eta(\tau)|^{2 D}$ is modular-invariant. In addition,

$$
\lim _{\tau_{2} \rightarrow \infty} F_{D}(\tau)=1
$$

since $\lim _{\tau_{2} \rightarrow \infty} \Theta(m, \tau)=1$.

It is convenient to define $W_{D}(\tau)=\tau_{2}^{D / 2} F_{D}(\tau)$. This function is modular-invariant, since multiplying by $\tau_{2}^{D / 2}$ cancels the modular weights of $F_{D}(\tau)$. Clearly $W_{D}(\tau) \sim \tau_{2}^{D / 2}$ for $\tau_{2} \rightarrow \infty$. Finally the differential equation for $F_{D}(\tau)$ becomes

$$
\left(\Delta_{\mathcal{H}}+s(s-1)\right) W_{D}(\tau)=0, \quad s=D / 2 .
$$

Thus $W_{D}(\tau)$ is an eigenfunction of $\Delta_{\mathcal{H}}$ with the eigenvalue $-s(s-1)$, which is negative for $D>2$.

For $D>2$, the differential equation (2.24) has no nonzero solution that grows at infinity more slowly than $\tau_{2}^{D / 2}$. This fact was important in $[16,17]$. Indeed, any solution of the differential equation that grows more slowly than $\tau_{2}^{D / 2}$ is bounded by a constant times $\tau_{2}^{1-D / 2}$, and therefore (for $D>2$ ) is a square-integrable eigenfunction of the Laplacian with the negative eigenvalue $-s(s-1)$. But the Laplacian on any manifold, acting on square-integrable wavefunctions, is strictly non-negative.

\footnotetext{
${ }^{7}$ To say that a function $f$ has modular weights $(u, v)$ with $u-v \in \mathbb{Z}$ means that $f((a \tau+b) /(c \tau+d))=$ $(c \tau+d)^{u}(c \bar{\tau}+d)^{v} f(\tau)$ for $\left(\begin{array}{ll}a & b \\ c & d\end{array}\right) \in \operatorname{SL}(2, \mathbb{Z})$. For example, $\tau_{2}=\operatorname{Im} \tau$ has modular weights $(-1,-1)$. The function $|\eta(\tau)|^{2}$ has modular weights $(1 / 2,1 / 2)$, so $F_{D}(\tau)$ must have modular weights $(D / 2, D / 2)$ to ensure modular invariance of the partition function. The case $u-v \in \frac{1}{2}+\mathbb{Z}$ is more complicated and will appear later.
} 
A function that satisfies all of the necessary conditions is the non-holomorphic (real analytic) Eisenstein series ${ }^{8}$

$$
E_{s}(\tau)=\sum_{(c, d)=1} \frac{\tau_{2}^{s}}{|c \tau+d|^{2 s}}
$$

The sum is over pairs of relatively prime integers $c, d$, up to sign (that is, we do not distinguish $(-c,-d)$ from $(c, d))$. Alternatively, the sum is over all modular images of the function $\tau_{2}^{s}$, since a general element $\left(\begin{array}{ll}a & b \\ c & d\end{array}\right) \in \operatorname{SL}(2, \mathbb{Z})$ maps $\tau_{2}^{s}$ to $\tau_{2}^{s} /|c \tau+d|^{2 s}$. So the sum in equation (2.25) can be alternatively written as

$$
E_{s}(\tau)=\sum_{\gamma \in P \backslash \mathrm{SL}(2, \mathbb{Z})} \operatorname{Im}(\gamma \tau)
$$

where $P=\left\{\left(\begin{array}{ll}1 & n \\ 0 & 1\end{array}\right)\right\}$ is the subgroup of $\operatorname{SL}(2, \mathbb{Z})$, isomorphic to $\mathbb{Z}$, that leaves $\operatorname{Im} \tau$ invariant. It is straightforward to check that the coprime integers $(c, d)$ uniquely label elements of the coset $P \backslash \mathrm{SL}(2, \mathbb{Z})$. The sum in eq. (2.25) converges for $\operatorname{Re} s>1$, which in our application means $D>2$.

Since $E_{s}(\tau)$ is a sum over all of the modular images of $\tau_{2}^{s}$, it is modular-invariant. The function $\tau_{2}^{s}$ is easily seen to be an eigenfunction of $\Delta_{\mathcal{H}}$ with eigenvalue $-s(s-1)$; the same therefore is true of its modular images, and of $E_{s}(\tau)$. Finally, it is immediate that $E_{s}(\tau) \sim \tau_{2}^{s}$ for $\tau_{2} \rightarrow \infty$. Thus, $E_{D / 2}(\tau)$ satisfies all of the desired properties of $W_{D}(\tau)$. These functions must be equal, since their difference $W_{D}(\tau)-E_{D / 2}(\tau)$ grows at infinity more slowly than $\tau_{2}^{D / 2}$ and hence must vanish, as discussed earlier.

Finally, we get an explicit formula for the average of the genus 1 partition function over the Narain moduli space $\mathcal{M}_{D}$ :

$$
\left\langle Z_{\Sigma}(m, \tau)\right\rangle=\frac{E_{D / 2}(\tau)}{\tau_{2}^{D}|\eta(\tau)|^{2 D}} .
$$

The numerator and denominator are both modular-invariant.

\subsection{Gravitational interpretation of the formula}

Our next task is to provide a possible interpretation of this formula in terms of an exotic bulk theory that is dual to an average over Narain moduli space.

As discussed in the introduction, the starting point is to assume that the bulk partition function, for the case that the conformal boundary is a surface $\Sigma$ of genus 1 , should be expressed as a sum over handlebodies. Let us decompose the genus 1 surface $\Sigma$ as $S^{1} \times S^{1}$, where the first factor parametrizes "space," and the second factor parametrizes "Euclidean time." One particular handlebody $Y$ with boundary $\Sigma$ is obtained by filling in the first factor by a two-dimensional disc $\mathrm{D}_{2}$. Thus $Y \cong \mathrm{D}_{2} \times S^{1}$. This handlebody can be obtained by Wick rotating Lorentzian $\mathrm{AdS}_{3}$ to Euclidean time via $t \rightarrow i t_{E}$, where $t$ is the usual

\footnotetext{
${ }^{8}$ It is important to note that this real analytic Eisenstein series is different from the holomorphic Eisenstein series (which transforms with modular weight $(n, 0)$ ) that commonly appears in the theory of modular forms.
} 
global time coordinate, and then periodically identifying $t_{E}$. This handlebody is usually referred to as thermal AdS, since it is the one used to study thermal physics in an AdS background.

Any other handlebody with boundary $\Sigma$ is obtained from $Y$ by a modular transformation of the boundary. In other words, to construct a more general handlebody one takes some other decomposition of $\Sigma$ as $S^{1} \times S^{1}$, and fills in the first factor by a disc. These other handlebodies are thus labelled by elements of the modular group $\operatorname{SL}(2, \mathbb{Z})$. In fact, because the element $T^{n}=\left(\begin{array}{ll}1 & n \\ 0 & 1\end{array}\right) \in \mathrm{SL}(2, \mathbb{Z})$ does not generate a new handlebody, each handlebody is uniquely labelled by an element of the coset $P \backslash \mathrm{SL}(2, \mathbb{Z})$, where $P$ is the subgroup of triangular matrices generated by $T$. One simple example is the handlebody obtained from thermal AdS by an $S$ transform - this handlebody is obtained by filling in the "Euclidean time" circle, rather than the spatial circle. This handlebody is the Euclidean continuation of the BTZ black hole in AdS.

In [6], a Hamiltonian approach was used to evaluate the path integral of Einstein gravity on $\mathrm{D} \times S^{1}$. In this approach, the key step is to determine the spectrum of physical states that arise in quantization on the spatial manifold $\mathrm{D}$. The partition function on $\mathrm{D} \times S^{1}$ is then evaluated as a trace in that Hilbert space. In Einstein gravity, there are no bulk excitations; the only physical states in quantization on D are the "boundary gravitons," first described by Brown and Henneaux [27]. The proposal in [6] was that the path integral on $\mathrm{D} \times S^{1}$ simply equals the partition function of the Brown-Henneaux modes. In other words, $\mathrm{D} \times S^{1}$ is thermal AdS, and in three spacetime dimensions, the only excitations in thermal AdS are the Brown-Henneaux modes. The resulting formula for the gravitational path integral on $\mathrm{D} \times S^{1}$ was later confirmed by a direct 1-loop computation ${ }^{9}$ in Einstein gravity [28].

In the present context, instead of the boundary gravitons, we should discuss the boundary modes of the current algebra. In other words, instead of Einstein gravity, we are here considering a theory that is supposed to be approximated, in some sense, by the $\mathrm{U}(1)^{2 D}$ Chern-Simons theory

$$
I_{\mathrm{CS}}=\sum_{I, J} \frac{\Lambda_{I J}}{2 \pi} \int_{Y} A^{I} \wedge \mathrm{d} A^{J},
$$

where $\Lambda$ is an even integral unimodular form of signature $(D, D)$. Instead of boundary gravitons, the Chern-Simon theory, if treated as in [29], has chiral and anti-chiral boundary current algebras which are abelian (since in this case we are studying an abelian gauge theory) and are each of rank $D$ (because of the signature of the quadratic form). As explained in the introduction, this relation of the bulk Chern-Simons theory to the boundary current algebra was the rationale for introducing the Chern-Simons theory.

The partition function of the boundary current algebras is the same as the partition function of $D$ left- and right-moving chiral bosons, with zero-modes omitted. It is simply

$$
Z_{\mathrm{CS}}^{D \times S^{1}}=\frac{1}{|\eta(\tau)|^{2 D}}=|q|^{-D / 12} \prod_{n=1}^{\infty} \frac{1}{\left|1-q^{n}\right|^{2 D}} .
$$

\footnotetext{
${ }^{9}$ There can be no higher order corrections, since the energy and momentum of the boundary gravitons are uniquely determined by conformal invariance along the boundary.
} 
This can be interpreted as the thermal partition function of a gas of $D$ "boundary photons," in the same way that the gravity partition function was the thermal partition function of a gas of boundary gravitons. It is the vacuum character of $D$ copies of the $\mathrm{U}(1) \times \mathrm{U}(1)$ current algebra. This expression can be verified in a direct bulk computation in $\mathrm{U}(1)^{2 D}$ Chern-Simons theory, just as in the gravity case. This will be discussed in section 4 . One important feature to note is that we have not included any separate factors of $|q|$ in equation (2.29), aside from the factors of $q^{1 / 24}$ which are contained in the definition of $\eta(\tau)$. In a normal theory of gravity such a factor would come from the classical Einstein action of the saddle point. In the present case we have not included a separate Einstein-Hilbert term in the action since, as explained in the introduction, the boundary stress tensor is itself an element of the $\mathrm{U}(1)^{2 D}$ current algebra. The factor of $|q|^{-D / 12}$ in equation (2.29) comes entirely from the bulk Chern-Simons computation, as we will discover in section 4, and can be regarded as a one-loop contribution of bulk Chern-Simons theory to the effective cosmological constant. We note also that equation (2.29) is one-loop exact because, as in the gravity case, the form of the answer is entirely fixed by the structure of the $\mathrm{U}(1)^{2 D}$ current algebra.

Equation (2.29) is the result of the bulk path integral for one particular handlebody with boundary $\Sigma$. To get the full partition function we need to sum over all handlebodies. That is, we must compute

$$
Z^{\text {bulk }}=\sum_{\gamma \in P \backslash \operatorname{SL}(2, \mathbb{Z})} \frac{1}{|\eta(\gamma \tau)|^{2 D}} .
$$

This is a much more straightforward problem than the superficially similar problem that was treated in [6]. We simply write

$$
\frac{1}{|\eta(\tau)|^{2 D}}=\frac{1}{\tau_{2}^{D / 2}|\eta(\tau)|^{2 D}} \cdot \tau_{2}^{D / 2}
$$

The function $\tau_{2}^{D}|\eta(\tau)|^{2 D}$ is modular invariant. So summing over modular images does nothing to this function. Thus we just need the sum over modular images of the function $\tau_{2}^{D / 2}$. But this sum was already done in equation (2.26); it equals the real-analytic Eisenstein series $E_{D / 2}(\tau)$.

So given our assumptions, the result that comes from summing over handlebodies is

$$
Z^{\text {bulk }}=\frac{E_{D / 2}(\tau)}{\tau_{2}^{D / 2}|\eta(\tau)|^{2 D}},
$$

which as we learned in section 2.2 is equal to the average of the partition function of the boundary CFT over the moduli space $\mathcal{M}_{D}$.

We consider the generalization of this derivation to higher genus in section 3 .

\subsection{Adding more information about the CFT}

We have so far considered the Siegel-Weil formula only for the case that the lattice $\Lambda$ is even and unimodular, as well as integral. In number theory, this restriction would be 
considered slightly artificial; there is a Siegel-Weil formula for an arbitrary integer lattice. Here we will sketch how this generalization can arise in a variant of the problem that we have considered so far. We will not be as detailed as we were in the case of an even unimodular lattice. In particular, we will not try to provide proofs of the more general version of the Siegel-Weil formula that we will invoke, though it appears that the approach in appendix A can potentially be generalized.

Up to this point, we have considered a boundary CFT about which we know nothing except that it has central charges $(D, D)$ and left- and right-moving $\mathrm{U}(1)^{D}$ current algebras. We did not assume any knowledge about the dimensions of primary fields of this theory. Instead we averaged over all possibilities, getting an answer with a plausible gravitational interpretation.

We could instead input some knowledge about the spectrum of primary fields and average only over the remaining possibilities. It turns out that this leads to more general versions of the Siegel-Weil formula.

As a special case, let us suppose that we know that the CFT has a primary field for the current algebra of dimension $(1,0)$. The condition under which this occurs is as follows. The vector space $V=\Lambda \otimes_{\mathbb{Z}} \mathbb{R}$ has a metric of signature $(D, D)$ that comes from the intersection form on the lattice $\Lambda$ and does not depend on the CFT moduli. Once one specifies those moduli, the metric $G$ of the torus determines the dynamics of the CFT fluctuations and one gets a decomposition $V=V_{+} \oplus V_{-}$, where $V_{+}$and $V_{-}$are subspaces on which the intersection form is positive or negative definite and (with a suitable orientation convention) are respectively the spaces of left- and right-moving modes of the CFT. Generically, neither $V_{+}$nor $V_{-}$contain any points of the lattice $\Lambda$; both $V_{+} \cap \Lambda$ and $V_{-} \cap \Lambda$ are generically empty. The condition for the CFT to have a current algebra primary of dimension $(1,0)$ is that there should be a point $x \in V_{+} \cap \Lambda$ of length squared 2 (it does not matter if this length is computed using the indefinite signature metric of $\Lambda$ or the positive-definite metric of $V_{+}$; these coincide for vectors in $V_{+}$). The existence of this $(1,0)$ primary, along with a second one that is associated to the vector $-x$, which also lies in $V_{+} \cap \Lambda$, extends a $\mathrm{U}(1)$ subalgebra of the CFT current algebra to $\mathrm{SU}(2)$ at level 1 , which we denote as $\mathrm{SU}(2)_{1}$. (If we want to specify the level of the $\mathrm{U}(1)^{2 D}$ current algebra, we could call it $\mathrm{U}(1)_{\Lambda}^{2 D}$ and then the extended current algebra would be $\mathrm{SU}(2)_{1} \times \mathrm{U}(1)_{\Upsilon_{\perp}}^{2 D-1}$, where $\Upsilon_{\perp}$ is introduced momentarily. We will not use this notation because it is not clear that subtleties concerning the level of the abelian current algebra are meaningful in the present context. See section 4.4.)

Specifying the existence of such an $x$ reduces the CFT moduli space from $\mathcal{M}_{D}$ to a subspace $\mathcal{M}_{D, x}$. Roughly speaking, $\mathcal{M}_{D, x}=\mathrm{SO}(D-1, D ; \mathbb{Z}) \backslash \mathrm{SO}(D-1, D ; \mathbb{R}) / \mathrm{SO}(D-$ $1) \times \mathrm{SO}(D)$. However, one has to clarify what is meant by $\mathrm{SO}(D-1, D ; \mathbb{Z})$. Let $\Upsilon_{0}$ be the rank 1 sublattice of $\Lambda$ that is generated by $x$, and let $\Upsilon_{\perp}$ be its orthocomplement. The group $\mathrm{SO}(D-1, D ; \mathbb{Z})$ that appears in the definition of $\mathcal{M}_{D, x}$ is the automorphism group of $\Upsilon_{\perp}$.

$\Upsilon_{0}$ is even but not unimodular; its quadratic form is the $1 \times 1$ matrix 2 , so its discriminant is the determinant of that matrix, or 2. Likewise $\Upsilon_{\perp}$ has discriminant 2. In particular $\Lambda$ is not the tensor product $\Upsilon_{0} \times \Upsilon_{\perp} ; \Lambda$ has discriminant 1 , and $\Upsilon_{0} \times \Upsilon_{\perp}$ has discriminant 
$2 \times 2=4$. The relation between them is

$$
\Lambda=\Upsilon_{0} \otimes \Upsilon_{\perp} \oplus \Upsilon^{\prime}
$$

where $\Upsilon^{\prime}$ is a coset of $\Upsilon_{0} \otimes \Upsilon_{\perp}$. More specifically, if $y \in \Lambda$ is any vector whose inner product with $x$ is an odd integer, then $\Upsilon^{\prime}$ consists of vectors of the form $y+z, z \in \Upsilon_{0} \otimes \Upsilon_{\perp}$. The Siegel-Narain theta function has a corresponding decomposition

$$
\Theta(m, \tau)=\Theta_{\Upsilon_{0} \otimes \Upsilon_{\perp}}+\Theta_{\Upsilon^{\prime}}
$$

where $\Theta_{\Upsilon_{0} \otimes \Upsilon_{\perp}}+\Theta_{\Upsilon^{\prime}}$ are computed, respectively, by sums over lattice points in $\Upsilon_{0} \otimes \Upsilon_{\perp}$ and in $\Upsilon^{\prime}$.

If we consider left-moving modes to be holomorphic and right-moving ones antiholomorphic, then the momentum-winding sum of $\Lambda_{0}$ is a holomorphic theta function

$$
\theta(\tau)=\sum_{n \in \mathbb{Z}} q^{n^{2}}, \quad q=\exp (2 \pi \mathrm{i} \tau)
$$

Because $\Lambda_{0}$ is not unimodular, this function is not mapped to itself by modular transformations. Rather,

$$
\theta(-1 / \tau)=\sqrt{\frac{\tau}{2 \mathrm{i}}}(\theta(\tau)+\widetilde{\theta}(\tau)), \quad \tilde{\theta}(\tau)=\sum_{r \in \mathbb{Z}+1 / 2} q^{r^{2}}
$$

The functions $\theta$ and $\widetilde{\theta}$ are associated to the two characters of the chiral algebra $\mathrm{SU}(2)_{1}$.

Let $m^{\prime}$ be the CFT moduli that remain after we insist on the existence of the vector $x \in \Lambda \cap V_{+}$. In other words, $m^{\prime}$ are the Narain moduli of $\Upsilon_{\perp}$. The expansion of $\Theta\left(m^{\prime}, \tau\right)$ in terms of theta functions of $\Lambda_{0}$ and $\Lambda_{\perp}$ is

$$
\Theta\left(m^{\prime}, \tau\right)=\theta(\tau) \Theta_{\Upsilon_{\perp}}\left(m^{\prime}, \tau\right)+\widetilde{\theta}(\tau) \widetilde{\Theta}_{\Upsilon_{\perp}}\left(m^{\prime}, \tau\right)
$$

where $\Theta_{\Upsilon_{\perp}}\left(m^{\prime}, \tau\right)$ is the momentum-winding sum of the lattice $\Upsilon_{\perp}$, and $\widetilde{\Theta}_{\Upsilon_{\perp}}\left(m^{\prime}, \tau\right)$ is a second function into which this transforms under modular transformations. The two terms on the right hand side of (2.37) are associated to the two summands in eq. (2.33).

Thus, in order to compute an average CFT partition function, we need to average $\Theta_{\Upsilon_{\perp}}\left(m^{\prime}, \tau\right)$ and $\widetilde{\Theta}_{\Upsilon_{\perp}}\left(m^{\prime}, \tau\right)$ over $\mathcal{M}_{D, x}$. We will write $\left\langle Z_{\Sigma}\left(m^{\prime}, \tau\right)\right\rangle_{x}$ for the average of $Z_{\Sigma}\left(m^{\prime}, \tau\right)$ over $\mathcal{M}_{D, x}$. The Siegel-Narain formula for the lattice $\Upsilon_{\perp}$ expresses this average in terms of real-analytic Eisenstein series $E(\tau)$ and $\widetilde{E}(\tau)$ of weights $(-1 / 2,0)$ :

$$
\left\langle Z_{\Sigma}\left(m^{\prime}, \tau\right)\right\rangle_{x}=\frac{\theta(\tau) E(\tau)+\widetilde{\theta}(\tau) \widetilde{E}(\tau)}{\tau_{2}^{D / 2}|\eta(\tau)|^{2 D}} .
$$

The definition of a function of modular weight $(-1 / 2,0)$ (or more generally of modular weight $(u, v)$ with $\left.u-v \in \frac{1}{2}+\mathbb{Z}\right)$ is rather subtle. The simplest definition is simply to say that $E(\tau)$ and $\widetilde{E}(\tau)$ transform in such a way that the expression on the right hand side of eq. (2.38) is modular invariant. A definition rather along these lines (for holomorphic forms) is given in Chapter IV of [30]. $E(\tau)$ and $\widetilde{E}(\tau)$ are given by formulas similar to 
eq. (2.25), with an extra factor $1 /(c \tau+d)^{1 / 2}$ in the denominator on the right hand side; there are also some congruence conditions on $c$ and $d$, and one has to include some roots of unity in the sum to compensate for such factors in the modular transformations of the theta functions. Details are described in [30] for the case of holomorphic modular forms of half-integral weight.

Qualitatively, eq. (2.38) is in agreement with what we might expect in a bulk analysis along the lines of section 2.3. As already noted, the existence of a vector $x \in \Lambda \cap V_{+}$extends the left-moving current algebra from $\mathrm{U}(1)^{D}$ to $\mathrm{SU}(2)_{1} \times \mathrm{U}(1)^{D-1}$. The right-moving current algebra is still $\mathrm{U}(1)^{D}$. So the natural bulk Chern-Simons theory is $\mathrm{SU}(2)_{1} \times \mathrm{U}(1)^{2 D-1}$. The starting point in trying to compute a bulk partition function is to determine the partition function of a handlebody $D \times S^{1}$ by taking a trace in the Hilbert space associated with quantization on $D$. In a hypothetical bulk theory that can be approximated in some sense by Chern-Simons theory of $\mathrm{SU}(2)_{1} \times \mathrm{U}(1)^{2 D-1}$, the natural physical states in quantization on $D$ are the current algebra modes on the boundary, and the corresponding partition function is $\theta(\tau) /|\eta(\tau)|^{2 D}$. To derive this formula, one just needs to know that the partition function of a holomorphic or antiholomorphic $\mathrm{U}(1)$ current algebra is $1 / \eta(\tau)$ or $1 / \overline{\eta(\tau)}$, while the partition function of the vacuum module of holomorphic $\mathrm{SU}(2)_{1}$ current algebra is $\theta(\tau) / \eta(\tau)$.

To get an ansatz for the bulk partition function in this situation, we sum over modular images of $\theta(\tau) /|\eta(\tau)|^{2 D}$. Writing

$$
\frac{\theta(\tau)}{|\eta(\tau)|^{2 D}}=\frac{\theta(\tau) \tau_{2}^{D / 2}}{\tau_{2}^{D / 2}|\eta(\tau)|^{2 D}},
$$

where the denominator is modular-invariant, we see that have to sum over the modular images of $\theta(\tau) \tau_{2}^{D / 2}$. This will generate the numerator on the right hand side of eq. (2.38). The details are somewhat complicated because the modular transformation of $\theta(\tau)$ is somewhat complicated, so we will not attempt more detail.

We have considered the special case that a U(1) subgroup of the current algebra is extended to $\mathrm{SU}(2)$, but one can analyze in a similar way any assumed extension of the $\mathrm{U}(1)^{D} \times \mathrm{U}(1)^{D}$ current algebra. ${ }^{10}$ In general, averaging over the remaining moduli via the Siegel-Weil formula always gives a result that has a more or less plausible interpretation in terms of an exotic bulk theory of gravity. We will just describe the construction that leads to the holomorphic case of the Siegel-Weil formula. Suppose that $D$ is a multiple of 8 , so that positive-definite even integer unimodular lattices of rank $D$ exist. Let $\Lambda_{-}$ be such a lattice, and let us stipulate that the CFT moduli are such that $\Lambda \cap V_{-} \cong \Lambda_{-}$. This corresponds to a particular extension of the right-moving $\mathrm{U}(1)^{D}$ current algebra. For example, if $D=8$, there is only one choice for $\Lambda_{-}$, namely the $\mathrm{E}_{8}$ lattice, and $\mathrm{U}(1)^{8}$ is extended to $\mathrm{E}_{8}$ current algebra at level 1. With our stipulation that $\Lambda \cap V_{-} \cong \Lambda_{-}$, it follows that $\Lambda \cap V_{+}$is equal to a possibly inequivalent even integer unimodular lattice $\Lambda_{+}$of rank $D$. However, for $D>8$, there are multiple isomorphism classes of such lattices, and all

\footnotetext{
${ }^{10}$ The extension does not necessarily involve an enhanced symmetry group. For example, if we had assumed a vector $x \in \Lambda \cap V_{+}$with $x^{2}=2 r, r>1$, we would get $\mathrm{U}(1)$ current algebra at level $r$.
} 
isomorphism classes can appear. At the point in moduli space at which $\Lambda_{+}$appears, the CFT partition function is

$$
\frac{\Theta_{\Lambda_{+}}(\tau) \bar{\Theta}_{\Lambda_{-}}(\tau)}{|\eta(\tau)|^{2 D}}
$$

Here $\Theta_{\Lambda_{+}}$and $\Theta_{\Lambda_{-}}$are holomorphic theta functions associated to the lattices $\Lambda_{+}$and $\Lambda_{-}$; $\Theta_{\Lambda_{-}}$is complex-conjugated because we have assumed the $\Lambda_{-} \subset V_{-}$so that the $\Lambda_{-}$modes are right-moving.

In this situation, the only possible averaging is over the choice of even integer unimodular lattice $\Lambda_{+}$; there are finitely many possibilities, depending on $D .{ }^{11}$ The holomorphic case of the Siegel-Weil formula says that the average ${ }^{12}$ of $\Theta_{\Lambda_{+}}$over all possibilities is a holomorphic Eisenstein series of weight $D / 2$ :

$$
\mathcal{E}_{D / 2}(\tau)=\sum_{(c, d)=1} \frac{1}{(c \tau+d)^{D / 2}} .
$$

Thus the average partition function of this class of theories is

$$
\left\langle Z_{\Sigma}(\tau)\right\rangle_{\Lambda_{-}}=\frac{\mathcal{E}_{D / 2}(\tau) \bar{\Theta}_{\Lambda_{-}}(\tau)}{|\eta(\tau)|^{2}}
$$

The symbol \langle\rangle$_{\Lambda_{-}}$represents an average under the constraint $\Lambda \cap V_{-} \cong \Lambda_{-}$.

To interpret this result from a gravitational point of view, we start with a seed partition function on $D \times S^{1}$, which we take to be the partition function of the extended chiral algebra. In the present example, this is $\bar{\Theta}_{-}(\tau) /|\eta(\tau)|^{2 D}$. We write this as

$$
\frac{1}{\eta(\tau)^{D}} \frac{\bar{\Theta}_{-}(\tau)}{\bar{\eta}(\tau)^{D}}
$$

To simplify the remaining derivation, let us assume that $D$ is divisible by 24 and not just by 8. Then $\frac{\bar{\Theta}-(\tau)}{\bar{\eta}(\tau)^{D}}$ is modular-invariant, and so we just have to sum over the modular images of the function $1 / \eta(\tau)^{D}$. For $D$ a multiple of 24 , the subtle $24^{\text {th }}$ roots of unity that appear in the modular transformation of $\eta(\tau)$ disappear, and we have just

$$
\eta((a \tau+b) /(c \tau+d))^{D}=(c \tau+d)^{D / 2} \eta(\tau) .
$$

With this, we see immediately that the sum over modular images of $1 / \eta(\tau)^{D}$ is $\mathcal{E}_{D / 2}(\tau) / \eta(\tau)^{D}$. So the sum over modular images of the gravitational expression in eq. (2.43) does give the formula (2.42) for the average partition function. If we had assumed that $D$ is divisible by 8 but not necessarily by 24 , we would have reached the same result after analyzing and canceling some cube roots of unity.

In this discussion, we started with the Narain family of CFT's, based on an even integer unimodular lattice $\Lambda$. Upon assuming an enhancement of the chiral algebra, we

\footnotetext{
${ }^{11}$ The number of such lattices is finite, but grows rapidly with $D$. For example, at $D=48$ there are at least $10^{120}$ such lattices, although the number is not known exactly. At large $D$, the number of even integer unimodular lattices up to isomorphism grows like $D^{D^{2}}$.

${ }^{12}$ In this averaging, one weights the contribution of a given lattice $\Lambda_{+}$by the inverse of the order of its automorphism group.
} 
restrict to a sublattice of $\Lambda$. Such a sublattice automatically is still even and integer but possibly not unimodular. So the averaging involves the Siegel-Weil formula for a general even integer lattice. Alternatively, to study a family of spin CFT's, which depend on a spin structure on $\Sigma$, we could start with an integer lattice $\Lambda$ that is unimodular but not even. After assuming an enhancement of the chiral algebra, $\Lambda$ would be replaced by an integer sublattice that generically is neither even nor unimodular. So the averaging in this case would depend on the Siegel-Weil formula for a general integer lattice.

\section{Higher genus and disconnected boundaries}

\subsection{Higher genus}

We will now describe the Siegel-Weil formula at higher genus, and understand its interpretation in terms of our conjectured exotic theory of gravity. The higher genus CFT partition function is more complicated, in part because a surface $\Sigma$ of genus $g>1$ does not admit a flat metric, and hence in any explicit formula there is no way to avoid the conformal anomaly. We will therefore need to be more schematic.

The genus $g$ partition function of a CFT in the Narain family can be written as

$$
Z_{\Sigma}(m, \tau)=\frac{\Theta(m, \tau)}{\Phi}
$$

where $\Theta(m, \tau)$ comes from a momentum-winding sum and is the Siegel-Narain theta function in genus $g$, and $\Phi$ comes from the integral over oscillator modes. As before, $m$ denotes a point in the CFT moduli space $\mathcal{M}_{D} ; \tau$ now represents the whole set of moduli of $\Sigma$.

Since the denominator $\Phi$ is not sensitive to the CFT moduli, averaging over $\mathcal{M}_{D}$ means averaging $\Theta(m, \tau)$ over $\mathcal{M}_{D}$. This average is described again by a Siegel-Weil formula. Using the higher genus analog of the Siegel-Weil formula, ${ }^{13}$ the result can be written as

$$
\left\langle Z_{\Sigma}(m, \tau)\right\rangle=\frac{E_{D / 2}(\tau)}{(\operatorname{det} \operatorname{Im} \Omega)^{D / 2}\left|\operatorname{det}^{\prime} \bar{\partial}\right|^{D}},
$$

where $E_{D / 2}(\tau)$, to be described shortly, is an Eisenstein series of the group $\operatorname{Sp}(2 g) ; \Omega$ is the period matrix of $\Sigma$; and $\operatorname{det}^{\prime} \bar{\partial}$ is the determinant of the $\bar{\partial}$ operator of $\Sigma$, mapping functions to $(0,1)$-forms, with zero-modes removed. In the denominator on the right hand side of eq. (3.2), the factor $(\operatorname{det} \operatorname{Im} \Omega)^{D / 2}$ generalizes $\tau_{2}^{D / 2}$ in eq. (2.27), and $\left|\operatorname{det}^{\prime} \bar{\partial}\right|^{D}$ generalizes $|\eta(\tau)|^{2 D}$ in that formula.

Our main interest here, however, is the Eisenstein series that appears in the numerator. Let us first restate in an alternative way the definition of the real analytic Eisenstein series that we used in genus 1 . If $\Sigma$ has genus 1 , then the lattice $\Gamma \subset H_{1}(\Sigma, \mathbb{Z})$ is a copy of $\mathbb{Z}^{2}$. Any primitive rank 1 sublattice $\Gamma_{0} \subset \Gamma$ is a Lagrangian sublattice. ${ }^{14}$ Once we pick a basis of $\Gamma$, say by choosing an $A$-cycle $\mathcal{A}$ and a $B$-cycle $\mathcal{B}$ on $\Sigma, \Gamma_{0}$ can be specified by giving its

\footnotetext{
${ }^{13}$ In appendix A, we discuss the derivation of the Siegel-Weil formula at genus $g$, by generalizing the method presented in the previous section.

${ }^{14}$ Lagrangian sublattices were defined in the introduction; for the definition of a primitive lattice, see footnote 4 .
} 
generator, which is a linear combination $c \mathcal{A}+d \mathcal{B}$, with relatively prime integer coefficients $c, d$, up to sign. Hence the sum over such pairs in the definition (2.25) of the Eisenstein series can be interpreted as a sum over Lagrangian sublattices.

The genus $g$ analog of the Siegel-Weil formula similarly involves a sum over Lagrangian sublattices. Once we pick a Lagrangian sublattice $\Gamma_{0} \subset \Gamma$, it is possible to define $\operatorname{det} \operatorname{Im} \Omega$ without any additional choices. To do this, we first pick a set of $A$-cycles $\mathcal{A}^{i}$ that provide a basis of $\Gamma_{0}$, and a complementary set of $B$-cycles $\mathcal{B}_{j}$, the nonzero intersection pairings being $\left\langle\mathcal{A}^{i}, \mathcal{B}_{j}\right\rangle=\delta_{j}^{i}$. The homology classes of the $\mathcal{B}_{i}$ are not uniquely determined, but they are determined up to $\mathcal{B}_{i} \rightarrow \mathcal{B}_{i}+n_{i j} \mathcal{A}^{j}, n_{i j} \in \mathbb{Z}$. Then one picks a basis of holomorphic 1-forms $\omega_{k}$ with $\oint_{\mathcal{A}^{i}} \omega_{j}=\delta_{j}^{i}$, and defines the period matrix by $\Omega_{i j}=\oint_{\mathcal{B}_{i}} \omega_{j}$. A shift $\mathcal{B}_{i} \rightarrow \mathcal{B}_{i}+n_{i j} \mathcal{A}^{j}$ shifts the period matrix by $\Omega_{i j} \rightarrow \Omega_{i j}+n_{i j}$, without changing $\operatorname{Im} \Omega$. Replacing the chosen $\mathcal{A}^{i}$ by a different basis of the same lattice $\Gamma_{0}$ changes $\Omega$ to $P \Omega P^{\mathrm{tr}}$, where $P$ is an integer-valued matrix of determinant $\pm 1\left(P^{\mathrm{tr}}\right.$ is the transpose of $P$ ), without affecting $\operatorname{det} \operatorname{Im} \Omega$. So in short $\operatorname{det} \operatorname{Im} \Omega$ is well-defined once $\Gamma_{0}$ is chosen.

For a given Lagrangian sublattice $\Gamma_{0}$, let $\operatorname{det} \operatorname{Im} \Omega_{\Gamma_{0}}$ be the corresponding value of the determinant of the imaginary part of the period matrix. Then the definition of the Eisenstein series is

$$
E_{s}(\tau)=\sum_{\Gamma_{0}}\left(\operatorname{det} \operatorname{Im} \Omega_{\Gamma_{0}}\right)^{s}
$$

The sum runs over all Lagrangian sublattices. For $g=1, \Omega$ is the $1 \times 1$ matrix $\tau=\tau_{1}+\mathrm{i} \tau_{2}$, so det $\operatorname{Im} \Omega=\operatorname{Im} \Omega=\tau_{2}$. Hence (3.3) reduces for $g=1$ to the sum over modular images of $\tau_{2}^{s}$. This is the definition that we used in eq. (2.25), though in that case we wrote an explicit formula for the dependence of $\operatorname{Im} \tau$ on the choice of Lagrangian sublattice. It is possible to do the same for any $g$, and rewrite eq. (3.3) as a sum over modular images just as in eqs. (2.25) and (2.26). This version of eqn (3.3), where the Eisenstein series is written explicitly as a sum over $\operatorname{Sp}(2 g, \mathbb{Z})$, is given in equation (A.20).

Now we can explain the properties that a hypothetical bulk dual of the average over Narain moduli space should have in order to reproduce the result (3.2) for the average partition function. The bulk contributions to the path integral should be labeled by Lagrangian sublattices $\Gamma_{0}$. In terms of classical geometry, we might try to attribute these contributions to handlebodies with boundary $\Sigma$, since (for connected $\Sigma$ ) these are in natural correspondence with Lagrangian sublattices, as noted in the introduction. That viewpoint will not work well in the disconnected case, which we come to in section 3.2, so instead we will just say that the bulk contributions are labeled by Lagrangian sublattices. The bulk path integral for a given $\Gamma_{0}$ should be

$$
\frac{1}{\left|\operatorname{det}_{\Gamma_{0}}^{\prime} \bar{\partial}\right|^{D}}
$$

where we note that the determinant depends on $\Gamma_{0}$ because of the subtleties involved in treating the kernel and cokernel of $\bar{\partial}$. Writing this as

$$
\frac{1}{\left(\operatorname{det} \operatorname{Im} \Omega_{\Gamma_{0}}\right)^{D / 2}\left|\operatorname{det}_{\Gamma_{0}}^{\prime} \bar{\partial}\right|^{D}} \cdot\left(\operatorname{det} \operatorname{Im} \Omega_{\Gamma_{0}}\right)^{D / 2}
$$


where the denominator $\left(\operatorname{det} \operatorname{Im} \Omega_{\Gamma_{0}}\right)^{D / 2}\left|\operatorname{det}_{\Gamma_{0}}^{\prime} \bar{\partial}\right|^{D}$ actually does not depend ${ }^{15}$ on $\Gamma_{0}$, we see that to get the full partition function, we just need to sum $\left(\operatorname{det} \operatorname{Im} \Omega_{\Gamma_{0}}\right)^{D / 2}$ over the choice of $\Gamma_{0}$. But this sum is the definition of the Eisenstein series $E_{D / 2}(\tau)$, so if (3.4) is the appropriate formula for the contribution of a given $\Gamma_{0}$ to the path integral, then the sum over all $\Gamma_{0}$ will indeed reproduce the desired answer (3.2) for the average partition function.

Eq. (3.4) is a fairly plausible formula for the handlebody path integral in a theory in which the only physical degrees of freedom are the boundary current algebra modes, the analogs of the Brown-Henneaux modes for gravity. Such boundary current algebra modes correspond to $D$ left- and right-moving massless scalars that lack zero-modes, and eq. (3.4) is a natural candidate for the path integral for such fields. One can think of $\frac{1}{\left|\operatorname{det}_{\Gamma_{0}}^{\prime} \bar{\partial}\right|^{D}}$ as a particular conformal block for the $\mathrm{U}(1)^{2 D}$ current algebra. This conformal block can be characterized by saying that what is propagating through any one-cycle that represents a class in $\Gamma_{0}$ is the vacuum module of the current algebra. In section 4 , we do a direct gauge theory calculation that, under certain assumptions, exhibits $\frac{1}{\left|\operatorname{det}_{\Gamma_{0}}^{\prime} \bar{\partial}\right|^{D}}$ as a gauge theory partition function in the handlebody.

The justification to consider only handlebodies is thin, as acknowledged in the introduction, unless we assume that we are studying an exotic theory of gravity in which "manifolds with boundary $\Sigma$ " are classified entirely by the associated Lagrangian sublattice $\Gamma_{0} \subset H_{1}(\Sigma, \mathbb{Z})$.

One more remark may provide some background for our discussion of the case that the boundary is not connected. The hypothetical theory that we are discussing is not conventional gravity and does not have a conventional semi-classical limit. The closest analog is to consider $D$ to be large. For generic $\tau$ there will be one Lagrangian sublattice $\Gamma_{0}$ that maximizes det $\operatorname{Im} \Omega_{\Gamma_{0}}$. For large $D$, this particular Lagrangian sublattice then makes the dominant contribution in the definition (3.3) of the Eisenstein series $E_{D / 2}$. Other contributions are exponentially suppressed. Of course, as we vary $\tau$, there will be large $D$ phase transitions at which two Lagrangian sublattices exchange dominance.

We will mention one additional subtlety which appears when we work at finite $D$ rather than in the large $D$ limit. This can be seen by investigating the Eisenstein series $E_{D / 2}(\tau)$ which appears as the average of the Siegel-Narain theta function. At genus 1, we saw that the Eisenstein series diverged unless we took $D>2$; this reflected the fact that the integral over $\mathcal{M}_{D}$ of the Siegel-Narain theta function was divergent. It turns out that the genus $g$ version of the Eisenstein series diverges unless

$$
D<g+1 .
$$

This and other properties of $E_{D / 2}(\tau)$ are discussed in more detail in appendix A. As in the genus one case, this reflects a genuine divergence of the averaging over Narain

\footnotetext{
${ }^{15}$ The expression $1 /\left(\left(\operatorname{det} \operatorname{Im} \Omega_{\Gamma_{0}}\right)\left|\operatorname{det}_{\Gamma_{0}}^{\prime} \bar{\partial}\right|^{2}\right)^{D / 2}$ is actually the partition function, per unit volume in the target space, of a sigma-model with target $\mathbb{R}^{D}$. Thus in particular it does not depend on the choice of $\Gamma_{0}$. See for example $[31,32]$. We will not explore this rather subtle point here as our interest in the present paper is really in the numerator of the partition function.
} 
moduli space. At finite $D$, the average partition function diverges for sufficiently large $g$ : when this happens, the typical CFT lives "at the boundary" of $\mathcal{M}_{D}$. The result is that the hypothetical gravitational dual theory can compute relatively coarse averaged CFT observables - namely, the low genus partition functions which encode the average spectrum and low moments of the OPE coefficients - but fails to compute highly refined observables, such as the large $g$ partition functions which compute higher moments of the OPE coefficients.

This has interesting implications for the structure of non-perturbative effects in our theory of gravity. As noted earlier (and described in more detail in section 4), our bulk Chern-Simons theory is one-loop exact at all genus, and so accounts for all of the perturbative effects which arise in a large $D$ limit. The Eisenstein series then computes a set of non-perturbative corrections. However, we see that at finite $D$ this is still not enough, as this sum diverges at sufficiently large genus. This may hint that further non-perturbative effects are necessary in order to render the theory sensible at finite $D$, analogous to the "doubly non-perturbative effects" [1] which are necessary in JT gravity in order to render the theory sensible nonperturbatively. ${ }^{16}$

\subsection{Disconnected boundaries}

As explained in the introduction, one is particularly interested to know what is the outcome of the averaging procedure if $\Sigma$ is not connected.

Recall first that the period matrix $\Omega$ of a genus $g$ Riemann surface $\Sigma$ is a $g \times g$ symmetric complex-valued matrix whose imaginary part is positive-definite. In what follows, $\Omega$ always refers to a complex matrix with those properties. For genus $g>3$, it is not true that any such $\Omega$ is the period matrix of some $\Sigma$. In general, such an $\Omega$ is associated to a principally polarized abelian variety of rank $g$ which is not necessarily the Jacobian of any $\Sigma$.

However, all the formulas of section 3.1 make sense for an arbitrary $\Omega$, whether or not it is the period matrix of a Riemann surface. For example, the Siegel-Narain theta function $\Theta(m, \tau)$ is defined by a momentum-winding sum that depends on $\Sigma$ only through its period matrix $\Omega$. The only properties of $\Omega$ that are needed for this sum to make sense are that it is symmetric and has positive-definite imaginary part. ${ }^{17}$ (Positivity is needed for convergence of the momentum-winding sum.) To emphasize this, we could denote the theta function as $\Theta(m, \Omega)$ rather than $\Theta(m, \tau)$. Moreover, the Siegel-Weil formula for averaging over $m$ holds for an arbitrary $\Omega$, not necessarily the period matrix of any Riemann surface. In fact, in the mathematical literature it is not usual to restrict $\Omega$ to be a period matrix.

This being so, before discussing disconnnected surfaces, we might want to ask if we can find a physical interpretation of the Siegel-Weil formula for an $\Omega$ that is not associated to a Riemann surface. Can we generalize the question that we have been asking so that the answer will involve the more general case of the Siegel-Weil formula? We can, though this involves asking a question that is possibly less natural than the question that we have

\footnotetext{
${ }^{16}$ We note that, although they diverge, the relevant Eisenstein series can be formally defined by analytic continuation for $g>D+1$. This may aid in interpreting our results at finite $D$.

${ }^{17}$ Properties of the space of such matrices, as well as an explicit formula for the Siegel-Narain theta function, are given in appendix A.
} 
been asking so far about the average of the partition function. Let $C_{\alpha}, \alpha=1, \cdots, 2 g$ be loops in $\Sigma$ that represent a basis of $H_{1}(\Sigma, \mathbb{Z})$, and let $X^{p}, p=1, \cdots, D$ be the scalar fields of a Narain model. We add to the action a bilocal term

$$
\sum_{\alpha \beta p q} d_{\alpha \beta p q} \oint_{C_{\alpha}} \mathrm{d} X^{p} \oint_{C_{\beta}} \mathrm{d} X^{q}
$$

with arbitrary coefficients $d_{\alpha \beta p q}$. This has no effect on the set of classical solutions of the theory, and no effect on the quantum oscillations around a classical solution. But it changes the action of a classical solution. By suitably adjusting the coefficients, we can arrange so that the momentum-winding sum is $\Theta(m ; \Omega)$ for any desired $\Omega$. So averaging over $m$ in this situation will involve the Siegel-Weil formula for arbitrary $\Omega$. The reader may or may not consider this a compelling context for the more general Siegel-Weil formula.

Regardless, a special case of the fact that the Siegel-Weil formula holds for any $\Omega$ is that it holds for any $\Sigma$, connected or not. For example, suppose that $\Sigma$ is the disjoint union of two connected surfaces $\Sigma^{\prime}$ and $\Sigma^{\prime \prime}$, of genus $g^{\prime}$ and $g^{\prime \prime}$, and whose moduli we denote as $\tau^{\prime}$ and $\tau^{\prime \prime}$. Set $g=g+g^{\prime}$ and write $\tau$ for the whole collection of moduli $\tau, \tau^{\prime}$. For fixed $m \in \mathcal{M}_{D}$, the partition function on $\Sigma$ is a product:

$$
Z_{\Sigma}(m, \tau)=Z_{\Sigma^{\prime}}\left(m, \tau^{\prime}\right) Z_{\Sigma^{\prime \prime}}\left(m, \tau^{\prime \prime}\right)
$$

We want to average over $m$ and compute the connected correlation function $\left\langle Z_{\Sigma^{\prime}}\left(m, \tau^{\prime}\right) Z_{\Sigma^{\prime \prime}}\left(m, \tau^{\prime \prime}\right)\right\rangle_{c}$. The function that we need to average is, from eq. (3.1),

$$
\frac{\Theta\left(m, \tau^{\prime}\right) \Theta\left(m, \tau^{\prime \prime}\right)}{\Phi_{\Sigma^{\prime}} \Phi_{\Sigma^{\prime \prime}}}
$$

It is the numerator that has to be averaged, since only the numerator depends on $m$.

Let $\Gamma^{\prime}=H_{1}\left(\Sigma^{\prime}, \mathbb{Z}\right), \Gamma^{\prime \prime}=H_{1}\left(\Sigma^{\prime \prime}, \mathbb{Z}\right)$, and $\Gamma=\Gamma^{\prime} \oplus \Gamma^{\prime \prime}=H_{1}(\Sigma, \mathbb{Z})$. On $\Gamma$, there is an intersection pairing, which is simply the sum of the intersection pairings on $\Gamma^{\prime}$ and on $\Gamma^{\prime \prime}$. A Lagrangian sublattice $\Gamma_{0}$ of $\Gamma$ is a ${ }^{18}$ rank $g$ sublattice of $\Gamma$ on which the intersection pairing vanishes. Such a sublattice may be the direct sum of Lagrangian sublattices $\Gamma_{0}^{\prime} \subset \Gamma^{\prime}$ and $\Gamma_{0}^{\prime \prime} \subset \Gamma^{\prime \prime}$, in which case we will say that $\Gamma_{0}$ is decomposable. But this is not the only possibility. There is no problem to define a period matrix of $\Sigma$ associated to a Lagrangian sublattice that is not decomposable. We will work out an example shortly.

Let $\Omega^{\prime}$ and $\Omega^{\prime \prime}$ be the period matrices of $\Sigma^{\prime}$ and $\Sigma^{\prime \prime}$. Then the direct sum ${ }^{19}$

$$
\Omega=\left(\begin{array}{cc}
\Omega^{\prime} & 0 \\
0 & \Omega^{\prime \prime}
\end{array}\right),
$$

\footnotetext{
${ }^{18}$ Primitive, as in footnote 4 in the introduction.

${ }^{19}$ Let $\widehat{\Sigma}$ be a connected Riemann surface defined as the connected sum of $\Sigma^{\prime}$ and $\Sigma^{\prime \prime}$. In a limit that $\widehat{\Sigma}$ degenerates to the union of $\Sigma^{\prime}$ and $\Sigma^{\prime \prime}$ joined at a point, the period matrix of $\widehat{\Sigma}$ reduces to that of the disconnnected surface $\Sigma$ (eq. (3.10)). This fact actually gives one way to prove that the Siegel-Weil formula must apply to disconnected Riemann surfaces if it applies to connected ones. But this is not very helpful in understanding the geometric meaning of the averaged path integral on a disconnected manifold, because a generic handlebody with boundary $\widehat{\Sigma}$ is not related in a nice way to a three-manifold whose boundary is the disjoint union of $\Sigma^{\prime}$ and $\Sigma^{\prime \prime}$. The problem arises precisely in the interesting case of indecomposable Lagrangian sublattices (see below).
} 
which we define as the period matrix of $\Sigma$, is symmetric with positive-definite imaginary part, so we can apply the Siegel-Weil formula to the corresponding Siegel-Narain theta function. But this theta function is just a product:

$$
\Theta(m, \Omega)=\Theta\left(m, \Omega^{\prime}\right) \Theta\left(m, \Omega^{\prime \prime}\right)
$$

because the momentum-winding sum of a disjoint union of Riemann surfaces is just the product of the two separate momentum-winding sums. The right hand side is the function that we want to average in order to compute $\left\langle Z_{\Sigma^{\prime}}\left(m, \tau^{\prime}\right) Z_{\Sigma^{\prime \prime}}\left(m, \tau^{\prime \prime}\right)\right\rangle$, and the left hand side is the function that we know how to average using the Siegel-Weil formula. Applying the Siegel-Weil formula, we learn that

$$
\left\langle Z_{\Sigma^{\prime}}\left(m, \tau^{\prime}\right) Z_{\Sigma^{\prime \prime}}\left(m, \tau^{\prime \prime}\right)\right\rangle=\frac{E_{D / 2}\left(\tau^{\prime}, \tau^{\prime \prime}\right)}{\left(\left(\operatorname{det} \operatorname{Im} \Omega^{\prime}\right)^{D / 2}\left|\operatorname{det}^{\prime} \bar{\partial}_{\Sigma^{\prime}}\right|^{D}\right)\left(\left(\operatorname{det} \operatorname{Im} \Omega^{\prime \prime}\right)^{D / 2}\left|\operatorname{det}^{\prime} \bar{\partial}_{\Sigma^{\prime \prime}}\right|^{D}\right)} .
$$

The denominator in this formula is the product of the denominators in the usual expressions for $Z_{\Sigma^{\prime}}$ and $Z_{\Sigma^{\prime \prime}}$; as usual it only depends on the moduli $\tau^{\prime}, \tau^{\prime \prime}$, and not on the choices that are used to define the period matrices and determinants. The definition of the Eisenstein series is as usual

$$
E_{D / 2}\left(\tau^{\prime}, \tau^{\prime \prime}\right)=\sum_{\Gamma_{0}}\left(\operatorname{det} \operatorname{Im} \Omega_{\Gamma_{0}}\right)^{D / 2}
$$

The sum runs over all Lagrangian sublattices $\Gamma_{0} \subset \Gamma$, and $\Omega_{\Gamma_{0}}$ is the period matrix defined using $\Gamma_{0}$. If we restrict the sum to the decomposable case $\Gamma_{0}=\Gamma_{0}^{\prime} \oplus \Gamma_{0}^{\prime \prime}$, where the summands are Lagrangian sublattices of $\Gamma^{\prime}$ and $\Gamma^{\prime \prime}$, respectively, then the right hand side will reduce to $E_{D / 2}\left(\tau^{\prime}\right) E_{D / 2}\left(\tau^{\prime \prime}\right)$. When inserted in eq. (3.12), this will give the disconnected contribution to the correlation function. The connected correlator comes precisely from Lagrangian sublattices that are not decomposable.

To make this more concrete, we will describe an explicit example of an indecomposable Lagrangian sublattice $\Gamma_{0}$ and compute its contribution to the connected correlator. Let $\Sigma^{\prime}$ and $\Sigma^{\prime \prime}$ be Riemann surfaces of genus 1 , with respective modular parameters $\tau^{\prime}$ and $\tau^{\prime \prime}$. On $\Sigma^{\prime}$, we pick an $A$-cycle $\mathcal{A}^{\prime}$ and a $B$-cycle $\mathcal{B}^{\prime}$; on $\Sigma^{\prime \prime}$ we pick an $A$-cycle $\mathcal{A}^{\prime \prime}$ and a $B$-cycle $\mathcal{B}^{\prime \prime}$. The nonzero intersection numbers are

$$
\left\langle\mathcal{A}^{\prime}, \mathcal{B}^{\prime}\right\rangle=\left\langle\mathcal{A}^{\prime \prime}, \mathcal{B}^{\prime \prime}\right\rangle=1 .
$$

We also pick holomorphic differentials $\omega^{\prime}$ on $\Sigma^{\prime}$ and $\omega^{\prime \prime}$ on $\Sigma^{\prime \prime}$ normalized so that

$$
\oint_{\mathcal{A}^{\prime}} \omega^{\prime}=\oint_{\mathcal{A}^{\prime \prime}} \omega^{\prime \prime}=1, \quad \oint_{\mathcal{B}^{\prime}} \omega^{\prime}=\tau^{\prime}, \quad \oint_{\mathcal{B}^{\prime \prime}} \omega^{\prime \prime}=\tau^{\prime \prime} .
$$

We now want to pick a Lagrangian sublattice $\Gamma_{0}$. A sublattice generated by, for example, $\mathcal{A}^{\prime}$ or $\mathcal{B}^{\prime}$ along with $\mathcal{A}^{\prime \prime}$ or $\mathcal{B}^{\prime \prime}$ is decomposable. Instead we pick one generated by

$$
\mathcal{A}^{1}=\mathcal{A}^{\prime}-\mathcal{A}^{\prime \prime}, \quad \mathcal{A}^{2}=\mathcal{B}^{\prime}+\mathcal{B}^{\prime \prime} .
$$

The minus sign in the definition of $\mathcal{A}^{1}$ ensures that $\left\langle\mathcal{A}^{1}, \mathcal{A}^{2}\right\rangle=0$, so that $\mathcal{A}^{1}$ and $\mathcal{A}^{2}$ indeed generate a Lagrangian sublattice. For a dual pair of cycles, we can pick

$$
\mathcal{B}_{1}=\mathcal{B}^{\prime}, \quad \mathcal{B}_{2}=-\mathcal{A}^{\prime \prime} .
$$


This ensures that $\left\langle\mathcal{B}_{1}, \mathcal{B}_{2}\right\rangle=0$ and

$$
\left\langle\mathcal{A}^{i}, \mathcal{B}_{j}\right\rangle=\delta_{j}^{i}
$$

To compute the period matrix, we need holomorphic differentials $\omega_{i}$ with

$$
\oint_{\mathcal{A}^{i}} \omega_{j}=\delta_{j}^{i}
$$

These are

$$
\omega_{1}=\frac{\tau^{\prime \prime} \omega^{\prime}-\tau^{\prime} \omega^{\prime \prime}}{\tau^{\prime}+\tau^{\prime \prime}}, \quad \omega_{2}=\frac{\omega^{\prime}+\omega^{\prime \prime}}{\tau^{\prime}+\tau^{\prime \prime}}
$$

The period matrix will then be

$$
\Omega_{i j}=\oint_{\mathcal{B}_{i}} \omega_{j}
$$

So

$$
\begin{aligned}
& \Omega_{11}=\oint_{\mathcal{B}_{1}} \omega_{1}=\frac{\tau^{\prime} \tau^{\prime \prime}}{\tau^{\prime}+\tau^{\prime \prime}} \\
& \Omega_{12}=\oint_{\mathcal{B}_{1}} \omega_{2}=\oint_{\mathcal{B}_{2}} \omega_{1}=\frac{\tau^{\prime}}{\tau^{\prime}+\tau^{\prime \prime}} \\
& \Omega_{22}=\oint_{\mathcal{B}_{2}} \omega_{2}=-\frac{1}{\tau^{\prime}+\tau^{\prime \prime}} .
\end{aligned}
$$

Expanding in real and imaginary parts by $\tau^{\prime}=\tau_{1}^{\prime}+\mathrm{i} \tau_{2}^{\prime}, \tau^{\prime \prime}=\tau_{1}^{\prime \prime}+\mathrm{i} \tau_{2}^{\prime \prime}$, we find that

$$
\operatorname{Im} \Omega=\frac{\tau_{2}^{\prime}}{\left|\tau^{\prime}+\tau^{\prime \prime}\right|^{2}}\left(\begin{array}{cc}
\left|\tau^{\prime \prime}\right|^{2} & \tau_{1}^{\prime \prime} \\
\tau_{1}^{\prime \prime} & 1
\end{array}\right)+\frac{\tau_{2}^{\prime \prime}}{\left|\tau^{\prime}+\tau^{\prime \prime}\right|^{2}}\left(\begin{array}{cc}
\left|\tau^{\prime}\right|^{2} & -\tau_{1}^{\prime} \\
-\tau_{1}^{\prime} & 1
\end{array}\right),
$$

which is positive-definite, as expected. There is a simple result for $\operatorname{det} \operatorname{Im} \Omega$ :

$$
\operatorname{det} \operatorname{Im} \Omega=\frac{\tau_{2}^{\prime} \tau_{2}^{\prime \prime}}{\left(\tau_{1}^{\prime}+\tau_{1}^{\prime \prime}\right)^{2}+\left(\tau_{2}^{\prime}+\tau_{2}^{\prime \prime}\right)^{2}}
$$

The contribution of this particular Lagrangian sublattice to the Eisenstein series is

$$
(\operatorname{det} \operatorname{Im} \Omega)^{D / 2}=\left(\frac{\tau_{2}^{\prime} \tau_{2}^{\prime \prime}}{\left(\tau_{1}^{\prime}+\tau_{1}^{\prime \prime}\right)^{2}+\left(\tau_{2}^{\prime}+\tau_{2}^{\prime \prime}\right)^{2}}\right)^{D / 2} .
$$

Intuitively, one expects that in anything that one would call a semiclassical limit, connected correlators between different components of $\Sigma$ should be small. As remarked near the end of section 3.1, in the present context, the closest analog of a semiclassical limit is large $D$. In fact, the connected contribution that we have analyzed is exponentially small for large $D$ compared to the disconnected correlation function. To see this, note that eq. (3.24) implies a general upper bound

$$
\operatorname{det} \operatorname{Im} \Omega \leq \frac{1}{4}
$$

where the maximum is attained if and only if $\tau_{1}^{\prime}+\tau_{1}^{\prime \prime}=0, \tau_{2}^{\prime}=\tau_{2}^{\prime \prime}$. Therefore, the contribution to the Eisenstein series from this particular indecomposable sublattice is at most 
$\left(\frac{1}{4}\right)^{D / 2}$. However, there is always a decomposable Lagrangian sublattice whose contribution to the Eisenstein series is at least $\left(\frac{3}{4}\right)^{D / 2}$. Indeed, the contribution of the decomposable sublattice generated by $\mathcal{A}^{\prime}$ and $\mathcal{A}^{\prime \prime}$ is $\tau_{2}^{\prime D / 2} \tau_{2}^{\prime \prime D / 2}$. If $\tau^{\prime}, \tau^{\prime \prime}$ are in the usual fundamental domain for $\operatorname{SL}(2, \mathbb{Z})$, then $\tau_{2}^{\prime}, \tau_{2}^{\prime \prime} \geq \sqrt{3} / 2$, and the corresponding contribution to the Eisenstein series is $\tau_{2}^{\prime D / 2} \tau_{2}^{\prime \prime D / 2} \geq\left(\frac{3}{4}\right)^{D / 2}$. Even if $\tau^{\prime}$ and $\tau^{\prime \prime}$ are not in the usual fundamental domain, by acting on $\mathcal{A}^{\prime}$ and on $\mathcal{A}^{\prime \prime}$ with separate $\operatorname{SL}(2, \mathbb{Z})$ transformations that map $\tau^{\prime}$ and $\tau^{\prime \prime}$ into the usual fundamental domain, we find a different decomposable Lagrangian sublattice whose contribution is at least $\left(\frac{3}{4}\right)^{D / 2}$. So the connected contribution to the correlator that we have examined is smaller than the disconnected correlator by at least a factor of $3^{D / 2}$. One expects that all connected contributions are similarly exponentially suppressed for large $D$. The interpretation in terms of a hypothetical bulk dual theory would be that "manifolds" with "wormhole" connections between different boundary components have larger action (or at least smaller quantum path integrals) than disconnected "manifolds."

The connected correlator that we have analyzed has the interpretation of

$$
\left\langle\operatorname{Tr} \exp \left(\mathrm{i} \tau_{1}^{\prime} P-\tau_{2}^{\prime} H\right) \operatorname{Tr} \exp \left(\mathrm{i} \tau_{1}^{\prime \prime} P-\tau_{2}^{\prime \prime} H\right)\right\rangle,
$$

where the traces are taken in the CFT Hilbert space, and $H$ and $P$ are the CFT Hamiltonian and momentum operators. This correlator is a real-analytic function of $\tau_{1}^{\prime}, \tau_{2}^{\prime}, \tau_{1}^{\prime \prime}$, and $\tau_{2}^{\prime \prime}$. so it can be analytically continued to complex values of those variables, at least within certain limits. In particular, to get an analog of the "spectral form factor," we can set $\tau_{2}^{\prime}=\beta+\mathrm{i} t, \tau_{2}^{\prime \prime}=\beta-\mathrm{i} t$, where $\beta$ and $t$ are both real; $t$ is interpreted as a real time parameter. The limit of large $t$, keeping fixed $\beta, \tau_{1}^{\prime}, \tau_{1}^{\prime \prime}$ probes interesting properties of the spectrum, and has been investigated in detail in other models; for example see [33]. It is not difficult to calculate the contribution of the indecomposable sublattice $\Gamma_{0}$ to the spectral form factor. For $\Sigma^{\prime}, \Sigma^{\prime \prime}$ both of genus 1, the denominator in the formula (3.12) for the correlation function simplifies to $\left(\operatorname{Im} \tau^{\prime} \operatorname{Im} \tau^{\prime \prime}\right)^{D / 2}\left|\eta\left(\tau^{\prime}\right) \eta\left(\tau^{\prime \prime}\right)\right|^{D}$. Since $\eta(\tau)$ is holomorphic and $|\eta(\tau+1)|=|\eta(\tau)|$, it follows that, when we give $\tau_{2}^{\prime}, \tau_{2}^{\prime \prime}$ imaginary parts $\pm \mathrm{i} t,\left|\eta\left(\tau^{\prime}\right) \eta\left(\tau^{\prime \prime}\right)\right|$ is periodic in $t$ with period 1 . This periodicity simply reflects the fact that the energy differences between current algebra modes are integer multiples of $2 \pi$. The current algebra modes are unaffected by averaging over Narain moduli space, so after this averaging the correlation function retains the periodic factor $1 /\left|\eta\left(\tau^{\prime}\right) \eta\left(\tau^{\prime \prime}\right)\right|^{D}$. More interesting is the $t$ dependence of the averaged product of Narain theta functions. This is

$$
\frac{1}{\left(\tau_{2}^{\prime} \tau_{2}^{\prime \prime}\right)^{D / 2}} \sum_{\Gamma_{0}}\left(\operatorname{det} \operatorname{Im} \Omega_{\Gamma_{0}}\right)^{D / 2}
$$

In view of eq. (3.25), the contribution to this expression of the particular indecomposable Lagrangian sublattice that we considered is actually a positive constant independent of $t$. We expect that the full spectral form factor has a positive constant limit at $t \rightarrow \infty$, just like the particular contribution that we have evaluated. We note that, as emphasized in [33], for the spectral form factor to approach a non-zero constant at late time is a key signature of the discreteness of the spectrum; this discreteness is, in general, quite difficult to see in a quantum gravity computation. 
One may ask for a geometric realization of the Lagrangian sublattice that we have considered. It is actually not difficult to find one. We want an oriented three-manifold $Y$ whose boundary consists of the disjoint union of $\Sigma^{\prime}$ and $\Sigma^{\prime \prime}$, such that $\mathcal{A}^{\prime}-\mathcal{A}^{\prime \prime}$ and $\mathcal{B}^{\prime}+\mathcal{B}^{\prime \prime}$ are boundaries in $Y$. We can take $Y=S^{1} \times S^{1} \times I$, where $I$ is a unit interval. The two ends of $I$ correspond to the two boundaries of $Y$, each of which is a copy of $S^{1} \times S^{1}$. We identify $\Sigma^{\prime}$ and $\Sigma^{\prime \prime}$ with the two boundaries of $Y$ in such a way that $\mathcal{A}^{\prime}$ and $\mathcal{A}^{\prime \prime}$ are identified with the first factor of $S^{1} \times S^{1}$ (in the first and second boundary of $Y$, respectively) and $\mathcal{B}^{\prime}$ and $-\mathcal{B}^{\prime \prime}$ are similarly identified with the second factor. The reason for a minus sign in the statement about $\mathcal{B}^{\prime \prime}$ is that $Y$ has to be oriented and its orientation has to induce the orientations of the boundaries $\Sigma^{\prime}$ and $\Sigma^{\prime \prime}$ that were built into the statement $\left\langle\mathcal{A}^{\prime}, \mathcal{B}^{\prime}\right\rangle=\left\langle\mathcal{A}^{\prime \prime}, \mathcal{B}^{\prime \prime}\right\rangle=1$. (The need for compatible orientations was noted following eq. (1.2).) So the identification of $\Sigma^{\prime}$ and $\Sigma^{\prime \prime}$ with the boundaries of $Y$ has to involve a relative orientation reversal. We used an orientation-reversing map of $\Sigma^{\prime \prime}$ that maps $\left(\mathcal{A}^{\prime \prime}, \mathcal{B}^{\prime \prime}\right) \rightarrow\left(\mathcal{A}^{\prime \prime},-\mathcal{B}^{\prime \prime}\right)$. We have given the simplest example of a $Y$ that is associated to the Lagrangian sublattice that we considered, but there are infinitely many others. Since there appears to be no natural way to get the answer $(\operatorname{det} \operatorname{Im} \Omega)^{D / 2}$ from a sum over distinct $Y$ 's, it was suggested in the introduction that in the exotic theory of gravity that is dual to an average over Narain moduli space, there is not a well-defined distinction between different $Y$ 's that are associated to the same Lagrangian sublattice.

We conclude by noting that, as with the connected case described in the previous subsection, the Eisenstein series which computes the analog of the sum over geometries in our theory of gravity does not necessarily converge when $D$ is finite. For example, we could consider the $N^{t h}$ moment of the torus partition function:

$$
\left\langle Z_{\Sigma_{1}}\left(m, \tau_{1}\right) \ldots Z_{\Sigma_{N}}\left(m, \tau_{N}\right)\right\rangle
$$

where the $\Sigma_{i}$ are tori. The Eisenstein series converges only when $N<D-1$. Thus at finite $D$ our theory can successfully compute relatively coarse features of the spectrum of the CFT, namely those features encoded in low moments of $Z_{\Sigma}(m, \tau)$, but fails when $N$ is large compared to $D$. This may indicate the need to include further non-perturbative effects.

Perhaps some intuition may be gained in this case by thinking of $Z$ not as a random CFT partition function, but rather as a random $D \times D$ matrix (a reasonable analogy, since $Z$ comes from a random rank $D$ lattice). Only the first $D$ moments of such a matrix are independent of one another, and these first $D$ moments are the only data required to completely characterize the probability distribution. It may be that the gravitational theory can only be used to compute the "independent" pieces of data needed to characterize the distribution on the space of CFTs. Having specified these data, the higher order observables are then completely determined. This is reminiscent of the notion of gravitational null states appearing in [34] (see also [35]).

\subsection{Genus zero}

We started this paper in genus 1, skipping the basic case of a surface of genus 0. Here we will make amends for this omission. 
If $\Sigma_{0}$ is a surface of genus 0 , then $\Sigma_{0}$ has no complex moduli. Moreover, the partition function of the Narain CFT on $\Sigma_{0}$ is $Z_{\Sigma_{0}}(m)=1$, independent of $m$. So averaging $Z_{\Sigma_{0}}(m)$ over $m$ will not have any effect.

From the point of view of the Siegel-Weil formula, since $H_{1}\left(\Sigma_{0}, \mathbb{Z}\right)=0$, there is no nontrivial sum to be carried out in averaging $Z_{\Sigma_{0}}(m)$. The only Lagrangian sublattice of the zero lattice $H_{1}\left(\Sigma_{0}, \mathbb{Z}\right)$ is the zero lattice. Since "manifolds with boundary $\Sigma$ " in the theory under discussion correspond in general to Lagrangian sublattices of $H_{1}(\Sigma, \mathbb{Z})$, we conclude that in the case of a surface of genus 0 , there is only one "manifold with boundary $\Sigma_{0} . "$ The closest analog of this object in classical geometry would be a three-ball, but in classical geometry it is far from unique as an oriented manifold whose boundary is a surface of genus 0 .

Since $Z_{\Sigma_{0}}(m)$ is a constant, independent of $m$, there is no connected correlator between $Z_{\Sigma_{0}}(m)$ and $Z_{\Sigma^{\prime}}(m, \tau)$ for any other surface $\Sigma^{\prime}$. In terms of the Siegel-Weil formula, one would interpret this as follows. Let $\Sigma$ be the disjoint union of $\Sigma_{0}$ and $\Sigma^{\prime}$. Since $H_{1}(\Sigma, \mathbb{Z}) \cong$ $H_{1}\left(\Sigma^{\prime}, \mathbb{Z}\right)$, "manifolds with boundary $\Sigma$ " are in 1-1 correspondence with "manifolds with boundary $\Sigma^{\prime}$." It appears that if there really is an exotic gravitational theory with the properties suggested by the Siegel-Weil formula, then in this theory there is no "wormhole" connecting a genus 0 surface to anything else. Alternatively, it may be that in this theory of gravity, there is no notion of whether spacetime is connected and thus no way to say whether or not $\Sigma_{0}$ and $\Sigma^{\prime}$ are connected through the wormhole.

\section{Path integrals in gauge theory}

\subsection{Preliminaries}

In this section, we will compare the formulas of sections 2 and 3 to direct evaluation of a gauge theory partition function on a handlebody. This will be done by adapting formulas in [28], where a similar direct calculation was done for Einstein gravity.

Let us first recall that for U(1) gauge theory with the standard Maxwell action, the partition function is

$$
Z_{\text {Max }}=\frac{\operatorname{det}^{\prime} \Delta_{0}}{\left(\operatorname{det}^{\prime} \Delta_{1}\right)^{1 / 2}}
$$

Here $\Delta_{0}$ is the Laplacian acting on a field of spin 0 , and $\Delta_{1}$ is the Laplacian acting on a vector field or 1-form. With a standard gauge-fixing, the denominator comes from the path integral over the gauge field, and the numerator is the ghost determinant.

Now consider a gauge field with Chern-Simons action. First consider the case that the gauge group is $\mathrm{U}(1)$, with a single gauge field $A$, and the action on a three-manifold $Y$ is

$$
\frac{1}{2 \pi} \int_{Y} A \wedge \mathrm{d} A
$$

In the approach to quantization followed in [29], the gauge-fixing action is

$$
\frac{1}{2 \pi} \int_{Y} \mathrm{~d}^{3} x \sqrt{g}\left(\phi D_{i} A^{i}+\bar{c} D_{i} D^{i} c\right)
$$


where $c$ and $\bar{c}$ are ghost and antighost fields and $\phi$ is a scalar field that is a BRST partner of $\bar{c}$. The path integral for $c$ and $\bar{c}$ gives the usual ghost determinant $\operatorname{det}^{\prime} \Delta_{0}$. The kinetic operator acting on the bosonic fields $A, \phi$ can be regarded as the operator $L_{-}=* \mathrm{~d}+\mathrm{d} *$ acting on differential forms of odd degree. The corresponding path integral is $1 / \sqrt{\operatorname{det}^{\prime} L_{-}}$. But since $L_{-}^{2}$ is equivalent to the direct sum $\Delta_{0} \oplus \Delta_{1}$, we have $\operatorname{det}^{\prime} L_{-}=\left(\operatorname{det}^{\prime} \Delta_{0}\right.$. $\left.\operatorname{det}^{\prime} \Delta_{1}\right)^{1 / 2}$. So finally the relevant ratio of determinants for U(1) Chern-Simons theory is $\left(\operatorname{det}^{\prime} \Delta_{0}\right)^{3 / 4} /\left(\operatorname{det}^{\prime} \Delta_{1}\right)^{1 / 4}$.

The path integral of U(1) Chern-Simons theory is not just a product of determinants, as there is also a phase that involves an Atiyah-Patodi-Singer $\eta$-invariant [29]. However, in the present paper we are interested in a $\mathrm{U}(1)^{2 D}$ theory based on a lattice $\Lambda$ of signature $(D, D)$, and in this case, the phase cancels between modes on which the metric of $\Lambda$ is positive and modes on which it is negative. An even unimodular integer lattice $\Lambda$ of signature $(D, D)$ is actually simply the direct sum of $D$ copies of a rank 2 lattice with intersection form

$$
H=\left(\begin{array}{ll}
0 & 1 \\
1 & 0
\end{array}\right) .
$$

So the $\mathrm{U}(1)^{2 D}$ Chern-Simons theory based on $\Lambda$ is simply the product of $D$ decoupled copies of a $\mathrm{U}(1)^{2}$ theory with two gauge fields $A, B$ and action

$$
\frac{1}{2 \pi} \int_{Y} A \wedge \mathrm{d} B
$$

To the extent that the partition function of this theory can be calculated just by evaluating determinants, the determinants involved are the same ones as in the last paragraph but with double the multiplicity, giving

$$
Z_{\mathrm{CS}}=\frac{\left(\operatorname{det}^{\prime} \Delta_{0}\right)^{3 / 2}}{\left(\operatorname{det}^{\prime} \Delta_{1}\right)^{1 / 2}}
$$

This formula was first obtained by A. Schwarz [36], who also recognized that this particular ratio of determinants is the Reidemeister-Ray-Singer torsion (of a background flat connection, a trivial one in the case of expanding around $A=B=0$ ).

Eq. (4.6) is not the whole story for evaluation of the partition function of Chern-Simons theory. It is not correct to simply ignore the zero-modes. Zero-modes of $\Delta_{1}$ means that the classical solution about which we are expanding is part of a family, and zero-modes of $\Delta_{0}$ mean that the gauge group has an unbroken subgroup of positive dimension. In a full evaluation of the Chern-Simons path integral, we have to integrate over the space of all classical solutions (summing in general over its connected components) and divide by the volume of the unbroken gauge group. Moreover, we are interested in evaluating the path integral for the case that $Y$ is a hyperbolic three-manifold with non-empty conformal boundary $\Sigma$. In Chern-Simons theory, one usually requires a subtle analysis of the asymptotic behavior of the fields near the boundary, while in scalar field theory or Maxwell theory, one can just assume that perturbations vanish at infinity.

Nevertheless, we will simply evaluate the right hand side of eq. (4.6), for the case that $Y$ is a hyperbolic three-manifold with conformal boundary $\Sigma$. We do this using the 
formulas that were obtained in [28] as part of a similar calculation for Einstein gravity. These are formulas for the determinants of $\Delta_{0}$ and $\Delta_{1}$ in a space of perturbations that vanish at infinity. We will find that this procedure works in the sense that - at least if $\Sigma$ is connected and $Y$ is a handlebody - it gives the result that was needed in eq. (3.4) to provide a bulk dual to the average over Narain moduli space.

Ideally, one would like to do a more rigorous evaluation of the Chern-Simons path integral and compare it precisely to the average over Narain moduli space. There seem to be real obstacles to this, as we discuss in section 4.4. Ultimately, we do not know to what extent gauge theory can be used to construct a bulk theory that is dual to an average over Narain moduli space.

\subsection{Path integral on a handlebody}

We will evaluate determinants by a heat kernel method, as in [28]. The basic idea is that the determinant of an operator $\Delta$ is given by the following formula:

$$
-\log (\operatorname{det} \Delta)=\int_{0^{+}}^{\infty} \frac{d t}{t} \operatorname{Tr}\left(K_{t}\right)
$$

where $K_{t} \equiv e^{-t \Delta}$. In the case of interest, where $\Delta$ is a differential operator on a manifold $Y$, the trace is just an integral over $Y$, and $K_{t}$ can be found by solving the differential equation

$$
\left(\partial_{t}+\Delta\right) K_{t}=0
$$

which we will momentarily write in position space as an equation for the heat kernel. The advantage of this technique is that (4.8) is a linear differential equation to which one can apply the method of images. Thus by starting with $K_{t}$ on hyperbolic three-space $\mathbb{H}^{3}$, one can easily obtain $K_{t}$ on a general quotient $\mathbb{H}_{3} / \mathrm{G}$, where $\mathrm{G}$ is a discrete group of automorphisms of $\mathbb{H}^{3}$. For simplicity, we will assume that every element of $G$ other than the identity is of infinite order; this is so in many interesting examples, including the groups (called Schottky groups) which are such that $\mathbb{H} / G$ is a handlebody.

As a simple illustration of this technique, let us take our operator to be the scalar Laplacian $\Delta_{0} \equiv-\nabla^{2}$ on $\mathbb{H}_{3}$, acting on the space of functions. We then introduce the heat kernel $K_{t}^{\mathbb{H}_{3}}\left(x, x^{\prime}\right)$, which solves the equation

$$
\left(\nabla_{x}^{2}-\partial_{t}\right) K_{t}^{\mathbb{H}_{3}}\left(x, x^{\prime}\right)=0
$$

with the initial condition $K_{t}\left(x, x^{\prime}\right)=\delta\left(x, x^{\prime}\right)$ at $t=0$. The solution is

$$
K_{t}^{\mathbb{H}_{3}}\left(x, x^{\prime}\right)=\frac{e^{-t-\frac{d^{2}}{4 t}}}{(4 \pi t)^{3 / 2}} \frac{d}{\sinh d}
$$

where $d=d\left(x, x^{\prime}\right)$ is the geodesic distance between $x$ and $x^{\prime}$. The determinant of the 
operator $\Delta_{0}$ on $\mathbb{H}_{3}$ is then

$$
\begin{aligned}
-\log \left(\operatorname{det}^{\prime} \Delta_{0}\right) & =\int_{0}^{\infty} \frac{\mathrm{d} t}{t} \int_{\mathbb{H}_{3}} \mathrm{~d}^{3} x \sqrt{g}\left(K_{t}^{\mathbb{H}_{3}}(x, x)\right) \\
& =\operatorname{Vol}\left(\mathbb{H}_{3}\right) \int_{0}^{\infty} \frac{\mathrm{d} t}{t} \frac{e^{-t}}{(4 \pi t)^{3 / 2}} \\
& =\frac{1}{6 \pi} \operatorname{Vol}\left(\mathbb{H}_{3}\right) .
\end{aligned}
$$

In writing the second line we have set $d(x, x)=0$ and pulled out an overall factor of $\mathrm{Vol}\left(\mathbb{H}_{3}\right)$. The resulting integral over $t$ diverges, reflecting the usual one-loop ultraviolet divergence. In the final line we have regulated this divergence by defining the $t$ integral by analytic continuation, as a Gamma function with negative argument. The final result is proportional to $\mathrm{Vol}\left(\mathbb{H}_{3}\right)$, and can be interpreted as a one-loop contribution to the bulk cosmological constant.

To apply this to quotients of the form $\mathbb{H}_{3} / G$, we first use the method of images to determine the heat kernel

$$
K_{t}^{\mathbb{H}_{3} / \mathrm{G}}\left(x, x^{\prime}\right)=\sum_{\gamma \in \mathrm{G}} K_{t}^{\mathbb{H}_{3}}\left(x, \gamma x^{\prime}\right)
$$

The determinant of the operator $\Delta_{0}$ on $\mathbb{H}_{3} / \mathrm{G}$ is then found by integrating:

$$
\begin{aligned}
-\log \left(\operatorname{det}^{\prime} \Delta_{0}\right) & =\int \frac{\mathrm{d} t}{t} \int_{\mathbb{H}_{3} / \mathrm{G}} \mathrm{d}^{3} x \sqrt{g}\left(K_{t}^{\mathbb{H}_{3} / \mathrm{G}}(x, x)\right) \\
& =\frac{1}{6 \pi} \operatorname{Vol}\left(\mathbb{H}_{3} / \mathrm{G}\right)+\sum_{\substack{\gamma \in \mathrm{G} \\
\gamma \neq 1}} \int \frac{\mathrm{d} t}{t} \int_{\mathbb{H}_{3} / \mathrm{G}} \mathrm{d}^{3} x \sqrt{g} K_{t}^{\mathbb{H}_{3}}(x, \gamma x) .
\end{aligned}
$$

In writing the second line we have separated out the $\gamma=1$ term in the sum and computed the $t$ integral as before, again finding a one-loop contribution to the cosmological constant. To interpret the other term, note that each $\gamma$ can be thought of as an element of the fundamental group, and the heat kernel is just a simple function of the length $d(x, \gamma x)$ of the corresponding geodesic. The result is that equation (4.13) takes the general form of a trace formula - such as the Selberg or Gutzwiller trace formula - where the spectrum of a Hamiltonian is related to the lengths of classical orbits. In the present case, the spectrum of $\nabla^{2}$ is related to the lengths of bulk geodesics. This will be a general feature of all of our formulas.

The sum over $G$ is typically impossible to carry out exactly. It can, however, be simplified by separating out the sum over primitive elements of $\mathrm{G}$ : an element $\gamma \in \mathrm{G}$ is primitive if it cannot be written as a positive power of any other element of G. Each primitive element $\gamma$ generates a $\mathbb{Z}$ subgroup of $G$ which we call a primitive subgroup (a primitive subgroup has two generators, namely $\gamma$ and $\gamma^{-1}$ ). The sum over $G$ reduces to a sum over the set $\mathcal{P}$ of primitive subgroups along with a sum over $\mathbb{Z}$ for each such subgroup. We note that, since the quotient $\mathbb{H}_{3} / \mathbb{Z}$ is a solid torus, we can associate to each 
primitive element $\gamma$ the modular parameter $\tau_{\gamma}$ of the associated boundary torus. Writing $\gamma \in \mathrm{G} \subset \operatorname{PSL}(2, \mathbb{C})$ as a $2 \times 2$ matrix, the modular parameter satisfies

$$
2 \cos \pi \tau_{\gamma}=\operatorname{Tr} \gamma
$$

This formula does not determine the sign of $\tau_{\gamma}$, which we fix so that $\operatorname{Im} \tau_{\gamma}>0$. It fixes $\operatorname{Re} \tau_{\gamma} \bmod 1(\bmod 1$ and not $\bmod 2$, because lifting from $\operatorname{PSL}(2, \mathbb{C})$ to $\operatorname{SL}(2, \mathbb{C})$ means that the sign of the right hand side is ill-defined). But our subsequent formulas will be expressed in terms of $q_{\gamma}=e^{2 \pi i \tau_{\gamma}}$, which depends on $\operatorname{Re} \tau_{\gamma}$ only $\bmod 1$.

It is now possible to evaluate the integrals $\mathrm{d} t$ and $\mathrm{d}^{3} x$ in equation (4.13), and write the result as a sum over the set $\mathcal{P}$ of primitive subgroups. The result is ${ }^{20}$

$$
\operatorname{det}^{\prime} \Delta_{0}=\exp \left\{-\frac{\operatorname{Vol}\left(\mathbb{H}_{3} / \mathrm{G}\right)}{6 \pi}\right\} \prod_{\gamma \in \mathcal{P}}\left(\prod_{\ell, \ell^{\prime}=0}^{\infty}\left(1-q_{\gamma}^{\ell+1} \bar{q}_{\gamma}^{\ell^{\prime}+1}\right)\right)^{2}
$$

This is a sum over primitive subgroups, so we do not count $\gamma$ and $\gamma^{-1}$ separately.

Although we have only written the formula for a massless scalar, this procedure can be applied (with more work) to find analogous heat kernel expressions for higher spin fields. The primary difficulty is dealing with the various different tensor structures that appear in the heat kernel. We refer to [28] for detailed computations. We will need only the result for the determinant for the spin one Laplacian $\Delta_{\mu}{ }^{\nu}=-\delta_{\mu}{ }^{\nu} \nabla^{2}+R_{\mu}{ }^{\nu}$. In [28], it was found that ${ }^{21}$

$$
\operatorname{det} \Delta_{1}=\operatorname{det}^{\prime} \Delta_{0} \cdot \prod_{\gamma \in \mathcal{P}}\left(\prod_{\ell, \ell^{\prime}=0}^{\infty}\left(1-q_{\gamma}^{\ell} \bar{q}_{\gamma}^{\ell^{\prime}+1}\right)\left(1-q_{\gamma}^{\ell+1} \bar{q}_{\gamma}^{\ell^{\prime}}\right)\right)^{2} .
$$

(It appears that this formula is valid even if the operator $\Delta_{1}$ has zero-modes. In that case, the determinant on the left hand side of eq. (4.16) vanishes, and the infinite product on the right hand side also vanishes. This will be discussed in section 4.3.)

We can now assemble these results together to evaluate the expression (4.6), which formally is the one-loop determinant of Chern-Simons theory, expanded around the trivial flat connection:

$$
\frac{\left(\operatorname{det}^{\prime} \Delta_{0}\right)^{3 / 2}}{\left(\operatorname{det} \Delta_{1}\right)^{1 / 2}}=\exp \left\{-\frac{\operatorname{Vol}\left(\mathbb{H}_{3} / \mathrm{G}\right)}{6 \pi}\right\} \prod_{\gamma \in \mathcal{P}}\left(\prod_{n=1}^{\infty} \frac{1}{\left|1-q_{\gamma}^{n}\right|}\right)^{2} .
$$

Let us first consider this formula for the solid torus $\mathbb{H}_{3} / \mathbb{Z}$, where $G$ is generated by a single primitive element $\gamma$ with $\operatorname{Tr}(\gamma)=2 \cos \pi \tau$. In this particular case, there is only one term in the product on the right hand side of eq. (4.17), so this is the only case in which we can evaluate the product in a completely explicit way. Although the volume of $\mathbb{H}_{3} / \mathbb{Z}$ is divergent, we may regularize it using the standard procedures of holographic

\footnotetext{
${ }^{20}$ Intermediate steps in this derivation can be found in [28].

${ }^{21}$ This formula is not written in precisely this way in [28]. In that reference, $\operatorname{det} \Delta_{1} / \operatorname{det}^{\prime} \Delta_{0}$ is formally called $\operatorname{det}^{\prime} \Delta_{\perp}$, and eq. (4.16) is written as a formula for $\operatorname{det}^{\prime} \Delta_{\perp}$.
} 
renormalization. One begins by cutting off the volume integral near the boundary and introducing boundary counterterms which remove the divergence that appears as this cutoff is taken away. The result is not invariant under conformal transformations on the boundary, so requires a choice of metric on the boundary. With the usual flat metric on the torus, one finds

$$
\operatorname{Vol}\left(\mathbb{H}_{3} / \mathbb{Z}\right)=-\pi^{2} \operatorname{Im} \tau .
$$

The final result for our the one-loop determinant is ${ }^{22}$

$$
\frac{\left(\operatorname{det}^{\prime} \Delta_{0}\right)^{3 / 2}}{\left(\operatorname{det} \Delta_{1}\right)^{1 / 2}}=|q|^{-1 / 12} \prod_{n=1}^{\infty} \frac{1}{\left|1-q^{n}\right|^{2}}=\frac{1}{|\eta(\tau)|^{2}} \text {. }
$$

We note that this one loop determinant naturally gives the usual prefactor of $|q|^{-1 / 12}$, which in the boundary language is attributable to the negative Casimir energy of a free boson on a circle. In bulk gravity calculations, such a term would typically arise from the regularized Einstein-Hilbert action of $\mathbb{H}_{3} / \mathbb{Z}$. In our Chern-Simons computation, this term came for free from the one-loop contribution to the bulk cosmological constant. Equation (4.19) is appropriate for $D=1$; for general $D$, one simply raises both sides to the power $D$. This is the result for the bulk path integral that we needed (eq. (2.31)) for a bulk theory dual to the average over Narain moduli space.

For higher genus, it is not possible to write such explicit formulas. But remarkably, it is possible to show in general that if $\Sigma$ is a connected Riemann surface of genus $g$, and $Y$ is a handlebody with conformal boundary $\Sigma$, then making the same assumptions, the bulk path integral on $Y$ agrees with what is needed in eq. (3.4) for the average over Narain moduli space to be reproduced by a sum over handlebodies.

If $Y$ is a genus $g$ handlebody, then its fundamental group $\mathrm{G}$ is a free group on $g$ generators. Such a subgroup of $\operatorname{PSL}(2, \mathbb{C})$, acting on $\mathbb{H}_{3}$ in such a way that the quotient is a handlebody $Y$, is called a Schottky group. In this case, Zograf [43], with further developments by McIntyre and Takhtajan [45], proved the following "holomorphic factorization formula"

$$
\left(\frac{\operatorname{det}^{\prime} \widehat{\Delta}_{0}}{\operatorname{det} \operatorname{Im} \Omega}\right)^{-1 / 2}=e^{S_{L} / 24 \pi} \prod_{\gamma \in \mathcal{P}}\left(\prod_{n=1}^{\infty} \frac{1}{\left|1-q_{\gamma}^{n}\right|^{2}}\right)
$$

where $\widehat{\Delta}_{0}$ is the Laplacian of a non-compact scalar on the surface $\Sigma .^{23}$ The function $S_{L}$ is an appropriately defined Liouville action on the moduli space of Schottky groups, defined explicitly in [44], which plays the role of the $|q|^{-1 / 12}$ factor in the torus case. Indeed, this

\footnotetext{
${ }^{22}$ Similar Chern-Simons computations have appeared in the literature before [38].

${ }^{23}$ The two dimensional Laplacian $\widehat{\Delta}_{0}$ should not be confused with the three dimensional Laplacian $\Delta_{0}$ which appeared earlier; in this section we will denote two-dimensional operators with a hat to avoid confusion. We note that both $\operatorname{det}^{\prime} \widehat{\Delta}_{0}(\Omega)$ and $S_{L}$ are not conformally invariant, but rather transform with conformal anomalies that match in such a way that eq. (4.20) is conformally invariant. We also note that we have written (4.20) as a product over primitive subgroups $\mathcal{P}$ where $\gamma$ and $\gamma^{-1}$ are not counted separately. In the literature this formula is often written in a slightly different way as a product over distinct primitive conjugacy classes, so that $\gamma$ and $\gamma^{-1}$ are counted separately.
} 
Liouville action was proven by Kraznov [40] to be proportional to the regularized volume of $\mathbb{H}_{3} / \mathrm{G}$

$$
S_{L}=-4 \operatorname{Vol}\left(\frac{\mathbb{H}_{3}}{\mathrm{G}}\right)+\text { counterterms }
$$

With this result, we see that eq. (4.20) matches exactly the path integral of $\mathrm{U}(1) \times \mathrm{U}(1)$ Chern-Simons theory on a genus $g$ handlebody given in eq. (4.17). This includes the factor of $S_{L}$ that, in other contexts, would arise from a regularized Einstein-Hilbert action. Recalling the purely two-dimensional version of holomorphic factorization, $\operatorname{det}^{\prime} \widehat{\Delta}_{0}=$ $(\operatorname{det} \operatorname{Im} \Omega)\left|\operatorname{det}_{\Gamma_{0}}^{\prime} \bar{\partial}\right|^{2}$ (see for example [31,32]; here $\Gamma_{0}$ is the Lagrangian sublattice associated to the handlebody), we conclude that our bulk path integral reproduces precisely the desired factor of $\left|\operatorname{det}_{\Gamma_{0}}^{\prime} \bar{\partial}\right|^{-D}$.

\subsection{Disconnected boundaries}

Remarkably, rather similar relationships between bulk and boundary functional determinants continue to hold even when the boundary is disconnected. The case that is wellestablished in the literature is the case that $Y$ is topologically $\Sigma_{0} \times I$, where $\Sigma_{0}$ is a surface of genus $g>1$ and $I$ is an open interval. In this case, if $Y$ is geometrically a quotient $\mathbb{H}_{3} / \mathrm{G}$, then the conformal infinity of $Y$ consists topologically of two copies of $\Sigma_{0}$. Generically, these two copies, which we will call $\Sigma^{\prime}$ and $\Sigma^{\prime \prime}$, have different complex structures. In that case, the group $G$ is called quasi-Fuchsian. In the special case that the two complex structures are the same, G is called Fuchsian. Since the Fuchsian case is just a special case, we need not consider it separately.

For quasi-Fuchsian groups, McIntyre and Teo [39] showed that for $n>1$,

$$
\left(\frac{\operatorname{det}^{\prime} \widehat{\Delta}_{n}\left(\Sigma^{\prime}\right)}{\operatorname{Im} \operatorname{det} \widehat{\Omega}_{n}^{\prime}}\right)^{-1 / 2}\left(\frac{\operatorname{det}^{\prime} \widehat{\Delta}_{n}\left(\Sigma^{\prime \prime}\right)}{\operatorname{Im} \operatorname{det} \widehat{\Omega}_{n}^{\prime \prime}}\right)^{-1 / 2}=e^{\frac{6 n(n-1)+1}{24 \pi} S_{L}} \prod_{\gamma \in \mathcal{P}}\left(\prod_{m=n}^{\infty} \frac{1}{\left|1-q_{\gamma}^{m}\right|^{2}}\right) .
$$

Here $\widehat{\Delta}_{n}$ is the boundary Laplacian acting on a field of spin $n$, and $\Omega_{n}$ is a generalized period matrix, which reduces to the usual period matrix if $n=1$ or $n=0 .{ }^{24}$ The left hand side is now interpreted as a product of one-loop determinants for the two individual boundary theories, regarded as functions of their period matrices. The Liouville action $S_{L}$ appearing in this equation is again proportional to the regularized volume of $\mathbb{H}_{3} / G$, just as in eq. (4.21); this result was established for quasi-Fuchsian groups by Takhtajan and Teo [41].

If we could set $n=0$ in this formula, we would be in the same situation as in section 4.2. After again invoking eq. (4.17), we would conclude that the product of determinants on $Y$ agrees with what is needed to reproduce the correlation functions that we studied in

\footnotetext{
${ }^{24} \mathrm{~A}$ crucial feature of this formula - analogous to our use of the bulk geometry in section 3.2 to determine an indecomposable Lagrangian sublattice - is that one is not free to choose independently the bases of holomorphic cycles on the boundary surfaces in which $\Omega^{\prime}$ and $\Omega^{\prime \prime}$ are computed. The choice of bases must be related in a particular way which depends on the bulk geometry [39]. In this way the left hand side of this formula depends implicitly on the choice of bulk geometry, consistent with the fact that the right hand side depends on it.
} 
section 3.2 between disconnected boundary components (at least for the special case that the two boundaries have the same genus and the indecomposable Lagrangian sublattice considered is associated to a three-manifold that is topologically $\Sigma_{0} \times I$ ).

In fact, the formula (4.22) does not hold for $n=0$. The infinite product on the right hand side is divergent in that case. We will explain this in a moment, but first we will point out that this should not be a surprise from the point of view of the bulk ChernSimons theory. For the case that the conformal boundary of $Y$ is the disjoint union of two components $\Sigma^{\prime}$ and $\Sigma^{\prime \prime}$, the bulk operator $\Delta_{1}$ has a zero-mode. This zero-mode is pure gauge but it cannot be gauged away by a gauge transformation that is trivial at the boundaries of $Y$. Being pure gauge, this mode does not contribute to any local gaugeinvariant observable, but it contributes to a Wilson line that stretches between the two conformal boundaries. To demonstrate the existence of this zero-mode, one can proceed as follows. Consider a function $f$ that equals 0 on $\Sigma^{\prime}$ and equals 1 on $\Sigma^{\prime \prime}$. If such a function approaches its limiting boundary values sufficiently quickly, then

$$
I(f)=\int_{Y} \mathrm{~d}^{3} x \sqrt{g}|\nabla f|^{2}
$$

is finite. By minimizing $I$ within the given class of functions $f$, one can ensure that $\Delta_{0} f=0$. Then $A=\mathrm{d} f$ is a zero-mode of $\Delta_{1}$ and is square-integrable since $I(f)<\infty$. Because the operator $\Delta_{1}$ has this zero-mode, the left hand of eq. (4.16) vanishes, so we are led to expect that the right hand side will also vanish. Equivalently, the left and right hand sides of eq. (4.17) should both be divergent.

The divergence in the infinite product in eq. (4.22) - or equivalently eq. (4.17) - is easiest to see in the Fuchsian case, so we concentrate on that case. The Fuchsian case is the case that $\mathrm{G}$ sits inside a $\operatorname{PSL}(2, \mathbb{R})$ subgroup of $\operatorname{PSL}(2, \mathbb{C})$. In that case, $Y=\Sigma \times I$ has a symmetry that exchanges the two ends of $I$. The fixed point set of this symmetry is a totally geodesic embedded surface $\Sigma \cong \mathbb{H}_{2} / \mathrm{G} \subset Y$, and all closed geodesics in $Y$ actually lie in $\Sigma$. So the product in (4.22) reduces to a product over geodesics in $\Sigma$. The Selberg zeta function associated to $\mathrm{G}$ is defined as:

$$
Z_{\mathrm{G}}(s) \equiv \prod_{\gamma \in \mathcal{P}} \prod_{m=0}^{\infty}\left(1-q_{\gamma}^{m+s}\right)=\prod_{\gamma \in \mathcal{P}} \prod_{m=0}^{\infty}\left(1-e^{-(m+s) L(\gamma)}\right)
$$

where $L(\gamma)$ is the length of the geodesic associated to $\gamma$. We see that the product in (4.22) is $\left|Z_{\mathrm{G}}(1)\right|^{-2}$. But it is a standard result that $Z_{\mathrm{G}}(1)=0$. This vanishing is equivalent to a divergence $\log Z_{\mathrm{G}}(s) \rightarrow-\infty$ for $s \rightarrow 1$. The important contributions to $Z_{\mathrm{G}}(s)$ for $s$ near 1 come from $m=0$ and from very long primitive closed geodesics:

$$
\log Z_{\mathrm{G}}(s) \approx-\int \rho(L) e^{-s L} d L
$$

where $\rho(L)$ is the density of primitive closed geodesics with length $L$. For a compact Riemann surface, we have $\rho(L) \sim \frac{e^{L}}{L}$ at large $L$, leading to a logarithmic divergence in $\log Z_{\mathrm{G}}(1)$ and vanishing of $Z_{\mathrm{G}}(1)$. (We are reversing the usual logic here: the usual procedure is to prove first in a more direct way thst $Z_{\mathrm{G}}(1)=0$ and then use this to constrain the large $L$ behavior of $\rho(L)$.) 


\section{$4.4 \mathrm{U}(1)^{2 D}$ and $\mathbb{R}^{2 D}$ Chern-Simons theories}

The divergence that we have just encountered actually has a simple fix if we take seriously the idea that the bulk theory is a Chern-Simons theory of the gauge group $\mathrm{U}(1)^{2 D}$. The Wilson line that stretches between the two boundary components of $Y$ is really valued in the gauge group. Instead of getting an infinity from a zero-mode of $\Delta_{1}$, we should get a factor of the volume of the gauge group, which is finite for gauge group $\mathrm{U}(1)^{2 D}$. This tells us, then, that we should aim to replace eq. (4.16) and subsequent formulas with a formula in which the zero-mode of $\Delta_{1}$ is removed on the left hand side. To compensate for this, the heat kernel formulas will have to be modified, and the right hand side of eq. (4.16) would be replaced with a regularized version. Hopefully, there would then also be a regularized version of the McIntyre-Teo formula for $n=0$.

This particular argument will clearly not work if we assume that the bulk gauge group is $\mathbb{R}^{2 D}$ rather than $\mathrm{U}(1)^{2 D}$. In this case, the volume of the gauge group is infinite, and the zero-mode of $\Delta_{1}$ will really lead to a divergence.

Nonetheless, it seems to be problematical to take too seriously the idea that the bulk theory is a $\mathrm{U}(1)^{2 D}$ gauge theory. One reason, which does not depend on the assumed gauge group, was explained in the introduction: gauge theory does not tell us to sum over manifolds, and it certainly does not tell us to sum over a specific class of manifolds, such as handlebodies. But there is actually a more specific problem if we assume that the theory is a $\mathrm{U}(1)^{2 D}$ Chern-Simons theory based on an even integer unimodular lattice $\Lambda$.

The $\mathrm{U}(1)^{2 D}$ Chern-Simons theory based on such a lattice is actually completely trivial, in a very strong sense. As already explained, this theory is equivalent to $D$ copies of a $\mathrm{U}(1)^{2}$ theory with action

$$
\frac{1}{2 \pi} \int_{Y} A \mathrm{~d} B
$$

Triviality of this theory is a special case of a statement in [46] and was analyzed in considerable detail in [47]. Triviality means, first of all, that if $Y$ is an oriented three-manifold without boundary, then the partition function of the theory on $Y$ is equal to 1 . Second, if $\Sigma$ is a Riemann surface, then the Hilbert space $\mathcal{H}_{\Sigma}$ of the theory on $\Sigma$ is 1-dimensional, and contains a distinguished unit vector $\Psi$. Third, if $Y$ is any oriented three-manifold with boundary $\Sigma$, then the path integral on $Y$ produces the same vector $\Psi \in \mathcal{H}_{\Sigma}$. All of these statements immediately carry over to the $\mathrm{U}(1)^{2 D}$ theory based on an even integer unimodular lattice.

In the case that $Y$ is noncompact, with conformal boundary $\Sigma$, it does not follow from this that the $\mathrm{U}(1)^{2 D}$ Chern-Simons path integral on $Y$ is equal to 1 ; this depends on what behavior of the fields is assumed near the conformal boundary. What does follow, however, is that the Chern-Simons path integral on $Y$ depends only on $\Sigma$ and not on $Y$. This may be proved as follows. Let $Y_{0}$ be a cutoff version of $Y$ in which the boundary $\Sigma$ is placed at a finite distance rather than at infinity. And let $U$ be the product of $\Sigma$ with a semi-open interval $[0,1)$. Thus we can build $Y$ by gluing together $Y_{0}$ and $U$ along $\Sigma$. The dependence of the Chern-Simons path integral on $Y$ is entirely encoded in the vector in $\mathcal{H}_{\Sigma}$ that is produced by the path integral on $Y_{0}$. But this is the same vector $\Psi$, independent of $Y_{0}$. 
Thus no matter what assumption we make about the behavior of fields near the conformal boundary, the $\mathrm{U}(1)^{2 D}$ Chern-Simons path integral depends only on the boundary and not on the bulk geometry.

What is happening is that $\mathrm{U}(1)^{2 D}$ Chern-Simons places too strong an equivalence relation on manifolds. In this Chern-Simons theory, all manifolds with given boundary $\Sigma$ are equivalent. What we would like instead would be for all manifolds with boundary $\Sigma$ that induce the same Lagrangian sublattice of $\Gamma=H_{1}(\Sigma, \mathbb{Z})$ to be equivalent. It is interesting that we get something very close to this if we just replace $\mathrm{U}(1)^{2 D}$ by $\mathbb{R}^{2 D}$. It suffices here to consider the basic case $D=1$ with the two gauge fields $A, B$. The phase space of $\mathbb{R}^{2}$ Chern-Simons on $\Sigma$ is the tensor product $V=\mathbb{R}^{2} \otimes H^{1}(\Sigma, \mathbb{R})$, with a symplectic form that is the tensor product of the quadratic form $\Lambda$ on $\mathbb{R}^{2}$ and the intersection form on $H^{1}(\Sigma, \mathbb{R})$. An element of $V$ is just a pair $A_{0}, B_{0}$ of gauge fields on $\Sigma$ satisfying $\mathrm{d} A_{0}=\mathrm{d} B_{0}=0$, up to gauge equivalence. The natural gauge-invariant observables in this theory are of the form $\oint_{\gamma} A, \oint_{\gamma} B$, where $\gamma$ is a homotopically nontrivial closed loop in $\Sigma$. (As the gauge group is $\mathbb{R}^{2}$ rather than $\mathrm{U}(1)^{2}$, these expressions are gauge-invariant, with no need to exponentiate them.) Suppose that $\Sigma=\partial Y$ and let $\Psi \in \mathcal{H}_{\Sigma}$ be the vector produced by the path integral on $Y$. Let $\Gamma_{0}$ be the Lagrangian sublattice of $\Gamma$ corresponding to $Y$. We claim that for any loop $\gamma \in \Sigma$ whose homology class $[\gamma]$ is in $\Gamma_{0}$,

$$
\oint_{\gamma} A \cdot \Psi=\oint_{\gamma} B \cdot \Psi=0
$$

Indeed, the condition $[\gamma] \in \Gamma_{0}$ means that $\gamma$ is the boundary of some oriented two-manifold $C \subset Y$, whence $\oint_{\gamma} A \cdot \Psi=\int_{C} \mathrm{~d} A \cdot \Psi=0$, since $\mathrm{d} A=0$ by the equations of motion. Similarly $\oint_{\gamma} B \cdot \Psi=0$. The operators $\oint_{\gamma} A$ and $\oint_{\gamma} B$, for $[\gamma]$ in a Lagrangian sublatttice $\Gamma_{0}$, are a maximal set of commuting observables in this theory, and a state that they annihilate is uniquely determined by $\Gamma_{0}$, up to a constant multiple. Thus any two $Y$ 's that induce the same Lagrangian sublattice $\Gamma_{0}$ generate the same state $\Psi$, up to an overall constant. (These overall constants are not all equal to 1, and for some $Y$ 's, the constants in question are divergent because of the infinite volume of the assumed gauge group.)

We would have preferred to learn that any two $Y$ 's associated to the same Lagrangian sublattice determine precisely the same state. This might have been an approximation to a statement that in the theory that we are looking for, "manifolds with boundary $\Sigma$ " are entirely classified by Lagrangian submanifolds. However, the $\mathbb{R}^{2 D}$ Chern-Simons theory has come pretty close.

Hopefully by now it is apparent that each of $\mathrm{U}(1)^{2 D}$ and $\mathbb{R}^{2 D}$ have both virtues and vices as candidate gauge groups for a theory dual to the average over Narain moduli space.

\section{Acknowledgments}

We thank A. Venkatesh for explanations of matters related to the Siegel-Weil formula. We also thank S. Kachru, L. Takhtadjan, Yingkun Li, D. Marolf, H. Maxfield, A. McIntyre, G.W. Moore, L.P. Teo, and A. Wall for helpful comments. Research of AM is supported in 
part by the Simons Foundation Grant No. 385602 and the Natural Sciences and Engineering Research Council of Canada (NSERC), funding reference number SAPIN/00032-2015. Research of EW is supported in part by NSF Grant PHY-1911298.

\section{A Derivation of the Siegel-Weil formula at $D>1$ and $g>1$}

In this appendix we will describe in more detail the derivation of the Siegel-Weil formula. We will begin in section A.1 by reviewing the moduli space $\mathcal{M}_{D}$ of CFTs with $D$ compact free bosons. We will show that the torus partition function obeys the differential equation (2.20) which was needed in our derivation of the genus one version of the Siegel-Weil formula. To discuss the higher genus version of this formula, we will first need to review in section A.2 some facts about the geometry of Siegel upper half space. We will then discuss the derivation of the higher genus version of the Siegel-Weil formula in section A.3.

\section{A.1 Narain moduli space}

A sigma-model with $T^{D}$ target space can be described by angle-valued fields $X^{p}, p=$ $1, \ldots, D\left(X^{p} \cong X^{p}+2 \pi\right)$, with a metric $G_{p q}$ and two-form field $B_{p q}$. In this Narain family of conformal field theories, $G$ and $B$ are constants that represent the moduli of the theory. We will call this moduli space $\mathcal{M}_{D}$, and denote a point in $\mathcal{M}_{D}$ by $m$.

The action, on a Euclidean signature worldsheet with coordinates $\sigma^{\alpha}, \alpha=1,2$, flat metric $\delta_{\alpha \beta}$, and Levi-Civita tensor $\varepsilon^{\alpha \beta}$, is

$$
I=\frac{1}{4 \pi \alpha^{\prime}} \int \mathrm{d}^{2} \sigma\left(G_{p q} \delta^{\alpha \beta} \partial_{\alpha} X^{p} \partial_{\beta} X^{q}+\mathrm{i} B_{p q} \varepsilon^{\alpha \beta} \partial_{\alpha} X^{p} \partial_{\beta} X^{q}\right) .
$$

The marginal operator that describes small perturbations $\delta G_{p q}, \delta B_{p q}$ of $G$ and $B$ is

$$
\mathcal{O}=\left(\delta G_{p q} \delta^{\alpha \beta}+\mathrm{i} \delta B_{p q} \varepsilon^{\alpha \beta}\right) \partial_{\alpha} X^{p} \partial_{\beta} X^{q} .
$$

Similarly to the case $D=1$ discussed in section 2.1 , by computing the two-point function of $\mathcal{O}$ one can determine the Zamolodchikov metric:

$$
\mathrm{d} s^{2}=G^{m p} G^{n q}\left(\mathrm{~d} G_{m n} \mathrm{~d} G_{p q}+\mathrm{d} B_{m n} \mathrm{~d} B_{p q}\right) .
$$

This is also the metric of $\mathcal{M}_{D}$ as a locally homogeneous space. The Laplacian derived from this metric is

$$
\Delta_{\mathcal{M}_{D}}=-G_{m p} G_{n q}\left(\widehat{\partial}_{G_{m n}} \widehat{\partial}_{G_{p q}}+\widehat{\partial}_{B_{m n}} \widehat{\partial}_{B_{p q}}\right)-G_{m n} \widehat{\partial}_{G_{m n}}
$$

where $\widehat{\partial}_{G_{m n}}=\frac{1}{2}\left(1+\delta_{m n}\right) \frac{\partial}{\partial G_{m n}}$ and $\widehat{\partial}_{B_{m n}}=\frac{1}{2} \frac{\partial}{\partial B_{m n}}$.

On a torus $\Sigma$ with modular parameter $\tau=\tau_{1}+\mathrm{i} \tau_{2}$, the partition function of the model is $Z_{\Sigma}(m, \tau)=\Theta(m, \tau) /|\eta(\tau)|^{2}$ where $\Theta(m, \tau)$ is the Siegel-Narain theta function and the denominator does not depend on $m$. $\Theta(m, \tau)$ is a sum over integer-valued momenta $n_{p}$ and windings $w^{q}$, which we abbreviate as $\vec{n}$ and $\vec{w}$. Explicitly

$$
\Theta(m, \tau)=\sum_{\vec{n}, \vec{w}} Q(\vec{n}, \vec{w} ; m, \tau)
$$


with

$$
Q(\vec{n}, \vec{w} ; m, \tau)=\exp \left(-\frac{\pi \tau_{2}}{\alpha^{\prime}}\left(G^{p q} v_{p} v_{q}+G_{p q} w^{p} w^{q}\right)+2 \pi \mathrm{i} \tau_{1} n_{p} w^{p}\right)
$$

where

$$
v_{p}=\alpha^{\prime} n_{p}+B_{p q} w^{q} .
$$

A computation similar to the one in section 2.1 but somewhat longer reveals that

$$
\left(\Delta_{\mathcal{H}}-D \tau_{2} \partial / \partial \tau_{2}-\Delta_{\mathcal{M}_{D}}\right) Q=0
$$

Here $\Delta_{\mathcal{H}}$, introduced in eq. (2.16), is the Laplacian of the upper half plane. Since this equation is linear, $\Theta$ satisfies the same equation:

$$
\left(\Delta_{\mathcal{H}}-D \tau_{2} \partial / \partial \tau_{2}-\Delta_{\mathcal{M}_{D}}\right) \Theta=0
$$

The steps that go from this result to the Siegel-Weil formula were explained in section 2.2.

\section{A.2 Geometry of Siegel upper half space}

We now wish to consider the partition function of the same family of CFTs on a genus $g$ Riemann surface $\Sigma$ with period matrix $\Omega=\Omega_{i j}$, where $i, j=1, \ldots, g$.

This period matrix $\Omega$ is an element of Siegel upper half space $\mathcal{H}_{g}$, which is the space of complex, symmetric $g \times g$ matrices with positive definite imaginary part:

$$
\mathcal{H}_{g} \equiv\left\{\Omega_{i j}: \Omega_{i j}=\Omega_{j i}, \operatorname{Im} \Omega>0\right\} .
$$

Although not every element of $\mathcal{H}_{g}$ can be realized as the period matrix of a Riemann surface, both the Siegel-Narain theta function and relevant Eisenstein series are well-defined functions on $\mathcal{H}_{g}$. This makes the analysis much easier, as $\mathcal{H}_{g}$ is considerably simpler than the moduli space of Riemann surfaces. We will just need to review a few facts about $\mathcal{H}_{g}$.

To describe the symplectic group $\operatorname{Sp}(2 g, \mathbb{R})$, we introduce a vector space of row vectors

$$
v=\left(\begin{array}{lllll}
b_{1} & b_{2} & \cdots & b_{g} & a^{1} \\
a^{2} & \cdots & a^{g}
\end{array}\right),
$$

with matrix elements $a^{1}, a^{2}, \cdots, a^{g}$ and $b_{1}, b_{2}, \cdots, b_{g}$ and a symplectic form $\sum_{i=1}^{g} \mathrm{~d} b_{i} \mathrm{~d} a^{i}$. $\operatorname{Sp}(2 g, \mathbb{R})$ consists of matrices

$$
\gamma=\left(\begin{array}{ll}
A & B \\
C & D
\end{array}\right),
$$

constructed from $g \times g$ blocks $A, B, C, D$, that act on $v$ on the right $v \rightarrow v \gamma$. The condition that $\gamma$ preserves the symplectic form is

$$
A B^{t}=B A^{t}, C D^{t}=D C^{t}, A D^{t}-B C^{t}=1 .
$$

$\operatorname{Sp}(2 g, \mathbb{R})$ is the group of real-valued matrices that satisfy these conditions. To get $\operatorname{Sp}(2 g, \mathbb{Z})$, which is known as the Siegel modular group, we restrict $A, B, C, D$ to be integer-valued. Likewise we restrict $a^{i}, b_{j}$ to be integers, giving an integer lattice $\Gamma$ on which $\operatorname{Sp}(2 g, \mathbb{Z})$ acts. In our application, $\Gamma=H_{1}(\Sigma, \mathbb{Z})$. 
The group $\operatorname{Sp}(2 g, \mathbb{R})$ acts on $\mathcal{H}_{g}$, by

$$
\Omega \rightarrow \gamma \Omega \equiv(A \Omega+B)(C \Omega+D)^{-1} .
$$

The group $\operatorname{Sp}(2 g, \mathbb{Z})$ acts in a proper and discontinuous fashion on $\mathcal{H}_{g}$. The fundamental domain for this action is complicated (for example at $g=2$ its boundary is a union of 28 pieces) but has finite volume. The quotient $\mathcal{A}_{g} \equiv \operatorname{Sp}(2 g, \mathbb{Z}) \backslash \mathcal{H}_{g}$ is known as the Siegel modular variety. When $\Omega$ is the period matrix of a Riemann surface $\Sigma$, this $\operatorname{Sp}(2 g, \mathbb{Z})$ action can be thought of as acting on $H_{1}(\Sigma, \mathbb{Z})=\mathbb{Z}^{2 g}$.

It is convenient to divide the period matrix into its real and imaginary parts, as $\Omega=x+\mathrm{i} y$. Since $y_{i j}$ is positive-definite, it is invertible; we will denote its inverse as $y^{i j}$. The metric on Siegel upper half space

$$
\mathrm{d} s^{2}=y^{i j} y^{k l}\left(\mathrm{~d} y_{i k} \mathrm{~d} y_{j l}+\mathrm{d} x_{i k} \mathrm{~d} x_{j l}\right)
$$

is Hermitian and invariant under the action of $\operatorname{Sp}(2 g, \mathbb{R})$. The associated Laplace-Beltrami operator can be written as

$$
\Delta_{\mathcal{H}_{g}}=-y_{i k} y_{j l}\left(\widehat{\partial}_{x_{i j}} \widehat{\partial}_{x_{k l}}+\widehat{\partial}_{y_{i j}} \widehat{\partial}_{y_{k l}}\right)
$$

where $\widehat{\partial}_{x_{i j}}=\frac{1}{2}\left(1+\delta_{i j}\right) \frac{\partial}{\partial x_{i j}}$ and $\widehat{\partial}_{y_{i j}}=\frac{1}{2}\left(1+\delta_{i j}\right) \frac{\partial}{\partial y_{i j}}$. This Laplacian commutes with the $\operatorname{Sp}(2 g, \mathbb{R})$ action.

The imaginary part of the period matrix transforms as

$$
\operatorname{Im} \Omega=(C \bar{\Omega}+D)^{t} \operatorname{Im}(\gamma \Omega)(C \Omega+D)
$$

so that

$$
\operatorname{det} \operatorname{Im} \gamma \Omega=\frac{\operatorname{det} \operatorname{Im} \Omega}{|\operatorname{det}(C \Omega+D)|^{2}}
$$

One can show that

$$
\left(\Delta_{\mathcal{H}_{g}}+\left(g s^{2}-\frac{g(g+1)}{2} s\right)\right)(\operatorname{det} \operatorname{Im} \Omega)^{s}=0
$$

The Laplacian commutes with the action of $\operatorname{Sp}(2 g, \mathbb{Z})$, so the Eisenstein series

$$
E_{s}(\Omega) \equiv \sum_{\gamma \in P \backslash \operatorname{Sp}(2 g, \mathbb{Z})}(\operatorname{det} \operatorname{Im} \gamma \Omega)^{s}=(\operatorname{det} \operatorname{Im} \Omega)^{s} \sum_{\gamma \in P \backslash \operatorname{Sp}(2 g, \mathbb{Z})}|\operatorname{det}(C \Omega+D)|^{-2 s}
$$

is an eigenfunction of the Laplacian with the same eigenvalue. This is the Eisenstein series which appears in (3.2) as the average CFT partition function on a genus $g$ surface. In this equation we have defined the Siegel parabolic subgroup $P \equiv\left\{\left(\begin{array}{cc}A & B \\ 0 & D\end{array}\right) \in \operatorname{Sp}(2 g, \mathbb{Z})\right\}$; this is the subgroup of $\operatorname{Sp}(2 g, \mathbb{Z})$ which acts trivially ${ }^{25}$ on $|\operatorname{det} \operatorname{Im} \Omega|$. It follows that $E_{s}(\Omega)$ is invariant under the action of $\operatorname{Sp}(2 g, \mathbb{Z})$. We note that the sum diverges when $\operatorname{Re} s \leq \frac{g+1}{2}$. The Eisenstein series $E_{s}(\Omega)$ can be analytically continued from the region of convergence

\footnotetext{
${ }^{25}$ Here one has to know that $\operatorname{det} D= \pm 1$, as explained shortly.
} 
to define a meromorphic function in the whole complex $s$-plane, but its direct relation to an average over Narain moduli space only holds for $\operatorname{Re} s>\frac{g+1}{2}$.

In eq. (3.3), we defined the Eisenstein series in a seemingly different way as a sum over Lagrangian sublattices. The relation between the two definitions is as follows. First of all, the condition $b_{i}=0$ defines a particular Lagrangian sublattice $\Gamma_{0} \subset \Gamma$. The subgroup of $\operatorname{Sp}(2 g, \mathbb{Z})$ that leaves $\Gamma_{0}$ fixed is precisely the Siegel parabolic group $P$. So the sum over $P \backslash \operatorname{Sp}(2 g, \mathbb{Z})$ is precisely the sum over Lagrangian sublattices. Concretely, $\Gamma_{0}$ is spanned by row vectors $(0, \mathbf{a})$, where $\mathbf{a}=\left(a^{1}, a^{2}, \cdots, a^{g}\right)$ is a $g$-component row vector. For $\gamma=$ $\left(\begin{array}{ll}A & B \\ C & D\end{array}\right) \in \mathrm{Sp}(2 g, \mathbb{Z})$, we have

$$
(0, \mathbf{a}) \gamma=(\mathbf{a} C, \mathbf{a} D) .
$$

Thus $\gamma$ maps $\Gamma_{0}$ to a new Lagrangian sublattice spanned by vectors $(\mathbf{a} C, \mathbf{a} D)$ for arbitrary a. So the Eisenstein series can be written as a sum over the pairs $(C, D)$, subject to the equivalence relation $(C, D) \cong(U C, U D), U \in \mathrm{GL}(g, \mathbb{Z})$, which comes from the action of $P$.

In proving that $P$ is the automorphism group of the Lagrangian sublattice $\Gamma_{0}$, there is just one nontrivial point. It is immediate that an element $\gamma=\left(\begin{array}{ll}A & B \\ 0 & D\end{array}\right) \in P$ maps $\Gamma_{0}$ to itself, but for it to be an automorphism of $\Gamma_{0}$ (as an integer lattice), one needs det $D= \pm 1$. In fact, any element of the symplectic group has determinant 1, and for the block triangular matrix $\gamma$ we have $\operatorname{det} \gamma=\operatorname{det} A \operatorname{det} D$. Since $\operatorname{det} A$ and $\operatorname{det} D$ are integers, the fact that $\operatorname{det} \gamma=1$ implies that $\operatorname{det} A$ and $\operatorname{det} D$ are both \pm 1 . This condition is equivalent to $A$ and $D$ having integer-valued inverses, and thus belonging to $\operatorname{GL}(g, \mathbb{Z})$. Actually for $\gamma \in P$, $A$ and $D$ can be arbitrary elements of $\operatorname{GL}(g, \mathbb{Z})$, constrained by $A D^{t}=1$.

The group $P$ also contains matrices $\left(\begin{array}{ll}1 & B \\ 0 & 1\end{array}\right)$, where the only constraint on $B$ is that it is symmetric and integer-valued. Such group elements act on the period matrix by $\Omega \rightarrow \Omega+B$, thus shifting $\operatorname{Re} \Omega$ by an arbitrary symmetric integer-valued matrix, and leaving $\operatorname{Im} \Omega$ fixed.

\section{A.3 The average CFT partition function at genus $g$}

We now consider the sigma-model with $T^{D}$ target space on a Riemann surface of genus $g$. The partition function is a function of both the Narain moduli and the moduli of the Riemann surface. As in the torus case, the partition function is equal to a Siegel-Narain theta function times an oscillator contribution. The oscillator contribution is independent of $\mathcal{M}_{D}$, so will factor out when we average over $\mathcal{M}_{D}$.

The genus $g$ version of the Siegel-Narain theta function depends on both $m \in \mathcal{M}_{D}$ and the period matrix $\Omega \in \mathcal{H}_{g}$ of our Riemann surface, and can be written as

$$
\Theta(m, \Omega)=\sum_{\mathbf{n}, \mathbf{w} \in \mathbb{Z}^{g \times p}} Q(\mathbf{n}, \mathbf{w}, m, \Omega)
$$

where

$$
Q(\mathbf{n}, \mathbf{w}, m, \Omega) \equiv \exp \left\{-\frac{\pi y_{i j}}{\alpha^{\prime}}\left(G^{p q} v_{p}^{i} v^{j}{ }_{q}+G_{p q} w^{i p} w^{j q}\right)+2 \pi i x_{i j} n_{p}^{i} w^{j p}\right\}
$$


with

$$
v_{p}^{i}=\alpha^{\prime} n_{p}^{i}+B_{p q} w^{i q} .
$$

Note that the momentum $\mathbf{n}=n^{i}{ }_{p}$ and winding $\mathbf{w}=w^{i p}$ are now $g \times p$ matrices, with $i=1, \ldots, g$ and $p=1, \ldots, D$. The Siegel-Narain theta function is not modular invariant, but rather transforms under $\operatorname{Sp}(2 g, \mathbb{Z})$ transformations in such way that $(\operatorname{det} \operatorname{Im} \Omega)^{D / 2} \Theta(m, \Omega)$ is invariant. It will therefore be convenient to work with this combination of the determinant and the theta function.

The starting point for our derivation of the Siegel-Weil formula is the following differential equation for $Q$ :

$$
\left(\Delta_{\mathcal{H}_{g}}-\Delta_{\mathcal{M}_{D}}+\frac{g D(D-g-1)}{4}\right)\left((\operatorname{det} \operatorname{Im} \Omega)^{D / 2} Q(\mathbf{n}, \mathbf{w}, m, \Omega)\right)=0 .
$$

The derivation of this differential equation from our previous expressions for the Laplacians is somewhat lengthy. So we will just make a few comments on its derivation. The first is that our formulas for $\Delta_{\mathcal{H}_{g}}$ and $\Delta_{\mathcal{M}_{D}}$ are quite similar to one another. So it is perhaps not surprising that many of the terms which appear when $\Delta_{\mathcal{H}_{g}}-\Delta_{\mathcal{M}_{D}}$ acts on $Q$ directly cancel with one another. There are additional terms which come from (among other things) the piece of $\Delta_{\mathcal{M}_{D}}$ which is linear in $\widehat{\partial}_{G_{p g}}$ acting on $Q$, but these cancel against the terms that are linear in $\partial_{y_{i j}}$ acting on $(\operatorname{det} \operatorname{Im} \Omega)^{D / 2}$. This just leaves the terms where all of the derivatives in $\Delta_{\mathcal{H}_{g}}$ act on $(\operatorname{det} \operatorname{Im} \Omega)^{D / 2}$, which gives the constant term in (A.25), according to equation (A.19).

We now perform the sum over $\mathbf{n}$ and $\mathbf{w}$ to get

$$
\left(\Delta_{\mathcal{H}_{g}}-\Delta_{\mathcal{M}_{D}}+\frac{g D(D-g-1)}{4}\right)\left((\operatorname{det} \operatorname{Im} \Omega)^{D / 2} \Theta(m, \Omega)\right)=0 .
$$

We define $W(\Omega) \equiv(\operatorname{det} \operatorname{Im} \Omega)^{D / 2} F(\Omega)$, where

$$
F(\Omega)=\int_{\mathcal{M}_{D}} \Theta(m, \Omega) \mathrm{d} \mu(m)
$$

is the average lattice theta function. We may then use the fact that, for sufficiently large $D$, the integral $\int_{\mathcal{M}_{D}} \Delta_{\mathcal{M}_{D}} \Theta(m, \Omega) \mathrm{d} \mu(m)$ vanishes to conclude that

$$
\left(\Delta_{\mathcal{H}_{g}}+\frac{g D(D-g-1)}{4}\right) W(\Omega)=0 .
$$

The result is that $W(\Omega)$ is an $\operatorname{Sp}(2 g, \mathbb{Z})$ invariant function which obeys precisely the same eigenvalue equation as $E_{D / 2}(\Omega)$.

We will now take $D>g+1$, so that the eigenvalue of $\Delta_{\mathcal{H}_{g}}$ is negative. In this case $W(\Omega)$ and $E_{D / 2}(\Omega)$ must be equal. We explained the proof of this step for genus $g=1$ in section 2.2: one shows that the difference $E_{D / 2}(\Omega)-W(\Omega)$ is square-integrable, and therefore as an eigenfunction of the Laplacian with a negative eigenvalue, it must vanish. The proof for arbitrary $g$ is similar but technically more complicated. We will only provide a sketch. 
What makes the case of general $g$ more complicated is that there are different ways that $\Omega$ can go to infinity. Of course, the inequivalent possibilities are somewhat limited by the $\operatorname{Sp}(2 g, \mathbb{Z})$ symmetry. Because of the symmetry of shifting $\Omega$ by an arbitrary integer-valued symmetric matrix (see the final comment of section A.2), there is no meaningful notion of $\operatorname{Re} \Omega$ becoming large, and we can keep it fixed in the following discussion. Similarly, we do not have to worry about the possibility that an eigenvalue of $y=\operatorname{Im} \Omega$ becomes small (thus reaching the boundary of the Siegel upper half space $\left.\mathcal{H}_{g}\right)$; by an $\operatorname{Sp}(2 g, \mathbb{Z})$ transformation we can map any limiting behavior of $y$ to the possibility that $y$ is becoming large. However, there are different ways for $y$ to become large and we have to be careful about this.

Looking back to the lattice sum (A.22) that enters the definition of the Siegel-Narain theta function, we see that when $y$ becomes large, some contributions to the sum over the $g$-plets of integers $n_{p}^{i}$ and $w^{j q}$ are strongly suppressed. For example, if $y$ becomes large in a completely generic way, all of its eigenvalues becoming large, then all contributions are strongly suppressed unless $\mathbf{n}=\mathbf{w}=0$. At the other extreme, if $y_{11}$ becomes large while other matrix elements of $y$ remain fixed, then the surviving contributions in the lattice sum are those with $n^{1}{ }_{p}=w^{1 q}=0$, but no constraint on the other integers in the lattice sum. In general, there are $g$ essentially different ways for $y$ to go to infinity. An example of the $k^{\text {th }}$ possibility is that the large matrix elements of $y$ might be $y_{11}, y_{22}, \cdots, y_{k k}$. A more general way to describe this situation is to say that the $k^{\text {th }}$ possibility is that $y$ goes to infinity in such a way that the lattice sum in eq. (A.22) is reduced to a sum over $(g-k)$-plets of integers, for some $k \in\{1,2,3, \cdots, g\}$. In terms of Riemann surfaces, what is happening is that a genus $g$ surface is degenerating to a surface of genus $g-k$, with $k$ pairs of points glued together.

We will first consider the case that $k=g$, which we will describe by saying that $y$ is uniformly large. First let us look at the Eisenstein series (A.20). We see immediately see that for generic large $y$ (with fixed $x=\operatorname{Re} \Omega$ ), the contribution to $E_{s}(\Omega)$ with $C=0$ is $(\operatorname{det} \operatorname{Im} \Omega)^{s}$, while any other contribution is of order $1 /(\operatorname{det} \operatorname{Im} \Omega)^{s}$. (There is only one contribution with $C=0$, because the condition $C=0$ means that $\gamma \in P$, regardless of $D$.) So for uniformly large $y$,

$$
E_{s}(\Omega) \sim(\operatorname{det} \operatorname{Im} \Omega)^{s}+\mathcal{O}\left((\operatorname{det} \operatorname{Im} \Omega)^{-s}\right) .
$$

Now let us look at the definition of the Siegel-Narain theta function in eq. (A.22). With $y$ large and generic, all contributions to the lattice sum with nonzero integers $\mathbf{n}, \mathbf{w}$ are strongly suppressed. Thus the Siegel-Narain theta function reduces to $\Theta(m, \Omega)=1$. After averaging this over $m$, we get $F(\Omega)=1$, and hence $W(\Omega)=(\operatorname{det} \operatorname{Im} \Omega)^{D / 2}$, plus corrections that vanish when $y$ becomes uniformly large. So we have confirmed that in this region, $E_{D / 2}(\Omega)$ and $W(\Omega)$ coincide, modulo terms that vanish asymptotically. ${ }^{26}$

\footnotetext{
${ }^{26} \mathrm{~A}$ detail about these subleading terms might be puzzling at first. In eq. (A.29), we see that the subleading terms in $E_{D / 2}(\Omega)$ are power law suppressed when $y$ becomes uniformly large, while from eqs. (A.22) and (A.23), it may appear that contributions to the theta function with $\mathbf{n}$ or $\mathbf{w}$ nonzero are exponentially suppressed for uniformly large $y$. That last statement is true for fixed values of the Narain moduli $G, B$. However, we are really interested in averaging over these moduli to get $F(\Omega)$. In this averaging, it is possible for $G$ to be very large. For uniformly large $y$, the averaged theta function has contributions that decay like a power of $y$ that come from $\mathbf{n}, \mathbf{w} \neq 0$ but $G \sim y$. This gives power law suppressed contributions to the averaged theta function because the measure of Narain moduli space decays as a power of $G$ for large $G$.
} 
Finally we will discuss what happens when $y$ becomes large in a nonuniform fashion. For illustration, we consider the case $k=1$. The other cases are similar. For $k=1$, we may assume that the only large matrix element of $y$ is $y_{11}$. Looking back to eq. (A.20), we see that all contributions to $E_{s}(\Omega)$ are suppressed in this region except those with $C_{i 1}=0, i=1, \cdots, g$. Since one of the columns of $C$ vanishes, it follows that there is a nonzero $g$-component row vector $\mathbf{a}_{0}$ with $\mathbf{a}_{0} C=0$. We can choose $\mathbf{a}_{0}$ to be primitive. The Lagrangian sublattice $\Gamma_{0}$ associated to the pair $(C, D)$ then contains the vector $\left(0, \mathbf{a}_{0} D\right)$. Using the equivalence relation $(C, D) \cong(U C, U D), U \in \mathrm{GL}(g, \mathbb{Z})$, which does not affect the condition $C_{i 1}=0$, we can put $\mathbf{a}_{0} D$ in the form $\mathbf{a}=(1,0,0, \cdots, 0)$. Once we do this, $\Gamma_{0}$ contains the vector $(\mathbf{b}, \mathbf{a})$, with $\mathbf{b}=(0,0, \cdots, 0) . \Gamma_{0}$ is spanned by this vector together with a rank $g-1$ lattice $\Gamma_{0}^{\prime}$ of vectors $(\mathbf{b}, \mathbf{a})$ where $\mathbf{b}$ and $\mathbf{a}$ have vanishing first component: $\mathbf{b}=\left(0, b_{2}, \cdots, b_{g}\right)$ and $\mathbf{a}=\left(0, a^{2}, \cdots, a^{g}\right)$. But $\Gamma_{0}^{\prime}$ is just a Lagrangian sublattice of $\mathbb{Z}^{2 g-2}$. Thus for $y_{11}$ large, $E_{s}(\Omega)$ reduces to $(\operatorname{det} \operatorname{Im} \Omega)^{s}$ times a sum over Lagrangian sublattices of $\mathbb{Z}^{2 g-2}$, plus terms that vanish for $y_{11} \rightarrow \infty$. Now let us compare this to the averaged theta function. In making this comparison, we assume inductively that we already know that $E_{D / 2}(\Omega)=W(\Omega)$ for genus less than $g$, and we will prove that for genus $g, E_{D / 2}(\Omega)-W(\Omega)$ vanishes for $y_{11} \rightarrow \infty$. (As explained earlier, if $D>g+1$, it then follows that $E_{D / 2}(\Omega)=W(\Omega)$ in genus $g$.) For this, we just observe from eqs. (A.22) and (A.23) that for $y_{11} \rightarrow \infty$, the surviving contributions to the lattice sum that define the theta function are those with $n^{1}{ }_{p}=w^{1 p}=0$, so that this lattice sum reduces to a sum of the same form with $g$ replaced by $g-1$. By the inductive hypothesis, the average of this restricted sum is related to the Eisenstein series with $g$ replaced by $g-1 .^{27}$ So $W(\Omega)$ for genus $g$ agrees for $y_{11} \rightarrow \infty$ with $E_{D / 2}(\Omega)$. As in footnote 26, to compare terms in $E_{D / 2}(\Omega)$ and in $W(\Omega)$ that vanish for $y_{11} \rightarrow \infty$, one must take into account the behavior at large $G$, which gives the dominant correction at large $y_{11}$ on the CFT side.

Open Access. This article is distributed under the terms of the Creative Commons Attribution License (CC-BY 4.0), which permits any use, distribution and reproduction in any medium, provided the original author(s) and source are credited.

\section{References}

[1] P. Saad, S.H. Shenker and D. Stanford, JT gravity as a matrix integral, arXiv:1903.11115 [INSPIRE].

[2] J.L. Cardy, Operator Content of Two-Dimensional Conformally Invariant Theories, Nucl. Phys. B 270 (1986) 186 [INSPIRE].

[3] P. Kraus and A. Maloney, A cardy formula for three-point coefficients or how the black hole got its spots, JHEP 05 (2017) 160 [arXiv:1608.03284] [INSPIRE].

\footnotetext{
${ }^{27}$ From the point of view of conformal field theory, the meaning of this is that when a handle of a Riemann surface becomes very long (something which is equivalent conformally to the degeneration from genus $g$ to genus $g-1$ ), the dominant contribution to the partition function comes from the vacuum state flowing through the long handle. This contribution is the partition function of the same theory on a surface of genus $g-1$.
} 
[4] J. Cardy, A. Maloney and H. Maxfield, A new handle on three-point coefficients: OPE asymptotics from genus two modular invariance, JHEP 10 (2017) 136 [arXiv:1705.05855] [INSPIRE].

[5] S. Collier, A. Maloney, H. Maxfield and I. Tsiares, Universal dynamics of heavy operators in $\mathrm{CFT}_{2}$, JHEP 07 (2020) 074 [arXiv: 1912.00222] [INSPIRE].

[6] A. Maloney and E. Witten, Quantum Gravity Partition Functions in Three Dimensions, JHEP 02 (2010) 029 [arXiv:0712.0155] [INSPIRE].

[7] C.A. Keller and A. Maloney, Poincaré Series, 3D Gravity and CFT Spectroscopy, JHEP 02 (2015) 080 [arXiv: 1407.6008] [INSPIRE].

[8] N. Benjamin, S. Collier and A. Maloney, Pure Gravity and Conical Defects, JHEP 09 (2020) 034 [arXiv: 2004.14428] [INSPIRE].

[9] K.S. Narain, New Heterotic String Theories in Uncompactified Dimensions < 10, Phys. Lett. B 169 (1986) 41 [INSPIRE].

[10] K.S. Narain, M.H. Sarmadi and E. Witten, A Note on Toroidal Compactification of Heterotic String Theory, Nucl. Phys. B 279 (1987) 369 [InSPIRE].

[11] C.L. Siegel, Indefinite Quadratische Formen und Funktionentheorie. I, Math. Ann. 124 (1952) 17.

[12] C.L. Siegel, Indefinite Quadratische Formen und Funktionentheorie. II, Math. Ann. 124 (1952) 364.

[13] H. Maass, Lectures on Siegel's Modular Functions, available at http://www.math.tifr.res.in/ publ/ln/tifr03.pdf.

[14] A. Weil, Sur Certaines Groupes d'Opérateurs Unitaires, Acta Math. 111 (1964) 143.

[15] A. Weil, Sur la Formule de Siegel dans la Théorie des Groupes Classiques, Acta Math. 113 (1965) 1.

[16] M.B. Green and M. Gutperle, Effects of D-Instantons, Nucl. Phys. B 498 (1997) 195 [hep-th/9701093] [INSPIRE].

[17] M.B. Green and S. Sethi, Supersymmetry constraints on type IIB supergravity, Phys. Rev. D 59 (1999) 046006 [hep-th/9808061] [INSPIRE].

[18] S. Ashok and M.R. Douglas, Counting flux vacua, JHEP 01 (2004) 060 [hep-th/0307049] [INSPIRE].

[19] G.W. Moore, Computation Of Some Zamolodchikov Volumes, With An Application, arXiv: 1508.05612 [INSPIRE].

[20] R. Dijkgraaf, J.M. Maldacena, G.W. Moore and E.P. Verlinde, A Black hole Farey tail, hep-th/0005003 [INSPIRE].

[21] X. Yin, On Non-handlebody Instantons in 3D Gravity, JHEP 09 (2008) 120 [arXiv: 0711.2803] [INSPIRE].

[22] A. Castro, M.R. Gaberdiel, T. Hartman, A. Maloney and R. Volpato, The Gravity Dual of the Ising Model, Phys. Rev. D 85 (2012) 024032 [arXiv:1111.1987] [inSPIRE].

[23] C.-M. Jian, A.W.W. Ludwig, Z.-X. Luo, H.-Y. Sun and Z. Wang, Establishing strongly-coupled $3 D$ AdS quantum gravity with Ising dual using all-genus partition functions, JHEP 10 (2020) 129 [arXiv:1907.06656] [INSPIRE]. 
[24] M. Headrick, A. Maloney, E. Perlmutter and I.G. Zadeh, Rényi entropies, the analytic bootstrap, and 3D quantum gravity at higher genus, JHEP 07 (2015) 059 [arXiv: 1503.07111] [INSPIRE].

[25] N. Afkhami-Jeddi, H. Cohn, T. Hartman and A. Tajdini, Free Partition Functions and an Averaged Holographic Duality, to appear.

[26] J. Polchinski, String Theory. Vol. 1, Cambridge University Press, Cambridge U.K. (1998).

[27] J. Brown and M. Henneaux, Central Charges in the Canonical Realization of Asymptotic Symmetries: An Example from Three-Dimensional Gravity, Commun. Math. Phys. 104 (1986) 207 [InSPIRE].

[28] S. Giombi, A. Maloney and X. Yin, One-loop Partition Functions of 3D Gravity, JHEP 08 (2008) 007 [arXiv:0804.1773] [INSPIRE].

[29] E. Witten, Quantum Field Theory and the Jones Polynomial, Commun. Math. Phys. 121 (1989) 351 [INSPIRE].

[30] N. Koblitz, Introduction to Elliptic Curves and Modular Forms, Springer-Verlag, Heidelberg Germany (1984).

[31] G.W. Moore and P.C. Nelson, Absence of Nonlocal Anomalies in the Polyakov String, Nucl. Phys. B 266 (1986) 58 [INSPIRE].

[32] P.C. Nelson, Lectures on Strings and Moduli Space, Phys. Rept. 149 (1987) 337 [InSPIRE].

[33] J.S. Cotler et al., Black Holes and Random Matrices, JHEP 05 (2017) 118 [Erratum ibid. 09 (2018) 002] [arXiv:1611.04650] [INSPIRE].

[34] D. Marolf and H. Maxfield, Transcending the ensemble: baby universes, spacetime wormholes, and the order and disorder of black hole information, JHEP 08 (2020) 044 [arXiv:2002.08950] [INSPIRE].

[35] A. Maloney, Geometric Microstates for the Three Dimensional Black Hole?, arXiv: 1508.04079 [INSPIRE].

[36] A.S. Schwarz, The Partition Function of Degenerate Quadratic Functional and Ray-Singer Invariants, Lett. Math. Phys. 2 (1978) 247 [InSPIRE].

[37] X. Yin, Partition Functions of Three-Dimensional Pure Gravity, Commun. Num. Theor. Phys. 2 (2008) 285 [arXiv:0710.2129] [INSPIRE].

[38] M. Porrati and C. Yu, Kac-Moody and Virasoro Characters from the Perturbative Chern-Simons Path Integral, JHEP 05 (2019) 083 [arXiv: 1903.05100] [INSPIRE].

[39] A. Mcintyre and L.P. Teo, Holomorphic Factorization of Determinants of Laplacians Using Quasi-Fuchsian Uniformization, Lett. Math. Phys. 83 (2008) 41 [math/0605605].

[40] K. Krasnov, Holography and Riemann surfaces, Adv. Theor. Math. Phys. 4 (2000) 929 [hep-th/0005106] [INSPIRE].

[41] L.A. Takhtajan and L.-P. Teo, Liouville action and Weil-Petersson metric on deformation spaces, global Kleinian reciprocity and holography, Commun. Math. Phys. 239 (2003) 183 [math/0204318] [INSPIRE].

[42] E. D'Hoker and D.H. Phong, On Determinants of Laplacians on Riemann Surfaces, Commun. Math. Phys. 104 (1986) 537 [InSPIRE]. 
[43] P. Zograf, Liouville Action on Moduli Spaces and Uniformization of Degenerate Riemann Surfaces, Algebra i Analiz 1 (1989) 136 [Leningrad Math. J. 1 (1990) 941].

[44] L.A. Takhtajan and P. Zograf, On the Uniformization of Riemann Surfaces and on the Weil-Petersson metric on the Teichmüller and Schottky Spaces, Mat. Sb. (N.S.) 132(174) (1987) 304 [Math. USSR-Sb. 60 (1988) 297].

[45] A. McIntyre and L.A. Takhtajan, Holomorphic factorization of determinants of laplacians on Riemann surfaces and a higher genus generalization of kronecker's first limit formula, Analysis 16 (2006) 1291 [math/0410294] [INSPIRE].

[46] J.M. Maldacena, G.W. Moore and N. Seiberg, D-brane charges in five-brane backgrounds, JHEP 10 (2001) 005 [hep-th/0108152] [INSPIRE].

[47] E. Witten, $\mathrm{SL}(2, \mathbb{Z})$ action on three-dimensional conformal field theories with Abelian symmetry, in From Fields To Strings: Circumnavigating Theoretical Physics, M. Shifman eds., World Scientific, New York U.S.A. (2005), pg. 1173 [hep-th/0307041] [InSPIRE]. 